\title{
Modeling and analysis of the coupled dynamics of machine degradation and repair processes using piecewise affine stochastic differential equations
}

\author{
Hoang M. Tran ${ }^{\mathrm{a}, \mathrm{b}}$, Satish T.S. Bukkapatnam ${ }^{\mathrm{a}}$ \\ ${ }^{a}$ Department of Industrial and System Engineering, Texas AEM University, 3131 TAMU, College Station, TX 77843-3131, USA \\ ${ }^{b}$ School of Applied Mathematics and Informatics, Hanoi University of Science and Technology, No1 Dai Co Viet, Hai Ba Trung, \\ Hanoi, Vietnam
}

\begin{abstract}
This paper introduces an approach to model the coupled dynamics of recurring degradation and restoration processes that take place in real-world systems, such as manufacturing machines, in the form of nonlinear (piecewise affine) differential equations. Unlike previous methods, interactions between degradation and repair dynamics that influence downtime distributions in such manufacturing systems can be explicitly considered and dependencies beyond correlations between the time between failures (TBF) and the time to repair (TTR) can be captured. The periodic solutions of the model capture the progressive evolution of long time-scale failure and repair patterns. The distribution of short time-scale failure-repair cycles can be captured by providing a class of random perturbations to certain model parameters. We provide sufficient conditions for the existence and stability of the resulting nonlinear stochastic differential equation ( $n$-SDE) model solutions that mimic the breakdown and repair patterns observed in many real-world manufacturing systems, namely, fairly regular (periodic) large breakdown and repair cycles, interspersed with highly right skewed distributions of short cycles. We also define the basin of attraction for the periodic orbit. The n-SDE model was parametrized using real-world data sets acquired from an automotive manufacturing assembly line segment, and the model solutions were compared with actual observations of TBF and TTR patterns, as well as the performance of the process. Our approach reduces the computation time by about $25 \%$ when compared to a discrete-event simulation model, which uses conventional TBF and TTR distributions, implemented on a commercial platform. Experimental investigations also suggest that the model can capture the correlations and nonlinear coupled dynamics that exist in real-world operations among TBF and TTR, which are typically ignored in traditional approaches.
\end{abstract}

Keywords: piecewise affine; machine degradation and repair; basin of attraction

\section{Introduction}

Continuous flow models have been employed to capture the influence of recurring breakdown (degradation) and repair (regeneration) cycles on the dynamics of discrete entities pertinent to manufacturing, communication networks, and various physical and biological systems [1-3]. These models essentially treat the movement of parts, packets, or any discrete entity in a system as a fluid flow, expressed in the form of linear or nonlinear differential equations. This modeling approach facilitates a more straightforward characterization of complex system dynamic patterns, including instabilities, bifurcations, transient behaviors, and chaos, and can provide an effective balance between accuracy and speed compared to other modeling approaches (e.g., discrete-event simulation (DES) [4] and queuing network models $[5,6])$. Although a variety of large volumes of data, especially from plant floor information

Email addresses: tran@tamu.edu (Hoang M. Tran), satish@tamu.edu (Satish T.S. Bukkapatnam) 
systems, are becoming available, data-driven synthesis of fluid flow models, however, has received relatively less attention, particularly in the domain of manufacturing systems. Much of the pertinent earlier work in manufacturing has focused on applying these models to gain certain crucial qualitative insights into the plant-floor and enterpriselevel system characteristics [1,7-17]. Nonetheless, the following gaps remain in data-driven synthesis of fluid flow models for real-world manufacturing systems:

(1) It is noteworthy that whereas most of the behaviors, such as limit cycles (periodic steady state), intermittencies, bifurcations [18] emerging due to the complex nonlinear and stochastic dynamics of a manufacturing system have little role to play in conventional QN (temporal dynamics is assumed to be a stationary, usually $2^{\text {nd }}$ order process) and micro DES models (linearity and ergodicity assumptions can hold over such short time-scales), analysis of these behaviors is crucial for aggregate flow models. Earlier fluid flow models $[7,9,13]$ have considered a limited set of these behaviors. A majority of the complex behaviors [19] possible in manufacturing systems remain to be investigated.

(2) The coupling between degradation and repair processes, although known to exist in many real-world operations, has not received much attention thus far. In many manufacturing systems, significant auto- and crosscorrelations have been reported between time between failures (TBF) and time to repair (TTR) series. For example, the TTR and TBF for several of the machines in the manufacturing system studied in the present work exhibited significant cross- and auto-correlations (values of correlation coefficients varied over a 0.2-0.5 range). It has been noted that ignoring such dependencies in the simulations can lead to erroneous performance estimation [20, 21]. Several approaches have been reported to model these correlations: generalized exponential distributions [22], bivariate exponential distributions [23], three parameter renewal process approximation [20, 24], multiple degradation states $[15,16]$, complex repair policies [25-29], and phase-type distribution approximation [30], an indirect model based on monitoring the system performance using statistical process control [31] or a performance function [32]. However, incorporation of multimodal failure and repair time distributions, noted in the present work, has not been reported in the literature.

(3) Capturing machine breakdowns and repairs into the flow models has received some attention in the literature [33], including exponential failure and repair time distributions [34], general Markov chain or Markov process with a finite number of states $[8,11,15-17,35-38]$, and dynamic meta-models [39]. Pertinently, machine breakdowns, if modeled as discrete events introduce sharp transitions that make the numerical methods for solving fluid flow 
models computationally intractable. While this issue may present complexities similar to these of a DES, the tractability of simulations can be severely impeded whenever the aforementioned complex behaviors and coupled dynamics are considered in the model.

Towards surmounting these gaps, the present work addresses the derivation and analysis of fluid flow models in the form of nonlinear stochastic differential equations (n-SDEs) that capture the coupled dynamics- between degradation and restoration processes of complex multi-stage manufacturing systems. In particular, degradation in most of the automated assembly systems manifest as long time-scale, fairly regular process (occurring as a result of repeated, "cyclic" loading), as well as short time-scale degradations that occur in components that have steep degradation and restoration rates (short degradation and restoration cycles, such as human attention span, and component misfeeds). Knowing this coupled dynamics will provide more useful information on controlling purpose than knowing the dynamics of each process separately as other methods. It is shown that piecewise affine model structures can capture the salient nonlinear dynamic behaviors such as chaos and bifurcation while having advantage of simple formulation and computation [40,41]. This class of models have also been employed to study the stability, bifurcation and other dynamic characteristics of nonlinear processes, such as rig-coupled SD oscillators [40], non-smooth beams [42], gear-pair systems with backlash [43], 'Reid springs' with piecewise linear, nonlinear characteristics [44, 45], water column oscillators in nuclear power plants [46], nonlinear, bilinear, and elastoplastic oscillators [47-49], piecewise linear sampled control systems [50], degradation path of a single-unit system [51, 52], and degeneration - regeneration cycles of neurons $[53,54]$ and living cells $[55-59]$. In this paper, we show that the model with at most cubic constraint set on the parameters can yield (steady state) periodic solutions that can mimic a source of large time-scale breakdown and repair (equivalently, large TBF and TTR) processes (see Theorem 1 in Section 3), and closed form expressions for such large time-scale TBF and TTR can be derived in terms of the model parameters (see Theorem 2 in Section 3). This ability to capture dynamic behaviors allows for the nature of the couplings between TTR and TBF to be quantified. Short time-scale, frequent breakdown and repair processes can be captured by introducing a class of stochastic extension (see Theorem 6) to model parameters. We also investigate the local stability and the basin of attraction of these periodic orbits (Theorems 3-5). The theoretical results are validated using real-world data sets on TBF and TTR acquired from an automotive manufacturing assembly line [60]. The result suggested that the model can capture certain dynamical aspects of empirical data. 


\section{System description and problem formulation}

The dynamic system considered for the present investigation is an assembly line segment from a leading automotive manufacturer. It consisted of 18 stations of which 16 are located in tandem. One pair of stations is located in a parallel arrangement in the assembly line. This system is a type of simple $N$-stage manufacturing system and is described as follows. First, the change in the length of the buffer at the downstream of the $k^{\text {th }}$ machine/operation is given by $d L_{k} / d t=u_{k-1}(t)-u_{k}(t)$ where $u_{k}(t)$ is the throughput velocity of machine $k$ and $L_{k}$ is the buffer inventory level at time $t$. Conventionally, $u_{k}(t)$ is modeled using random processes $\mu_{k}(t)$ and $\nu_{k}(t)$ as

$$
u_{k}(t)=\mu_{k}(t)-\nu_{k}(t)
$$

where $\mu_{k}(t)$ is the processing rate (during the up time), $\nu_{k}(t)$ is the throughput rate loss due to degradation/ breakdown and, typically, $u_{k}(t)=0$ during downtime. Downtime in many manufacturing system operations may be attributed to the following three causes:

1. Machine breakdown and repair that takes place during times $t \in \mathbb{T}_{\text {repair }}^{k}$,

2. Starving (upstream buffer is empty) that takes place during times $t \in \mathbb{T}_{\text {starve }}^{k}$ and/or

3. Blocking (downstream buffer is full) that takes place during times $t \in \mathbb{T}_{\text {block }}^{k}$, i.e.,

$$
u_{k}(t)=0 \text { if }\left(L_{k}(t)=0 \text { or } L_{k+1}=L_{k+1}^{\max } \text { or } t \in \mathbb{T}_{\text {repair }}^{k}\right)
$$

Our problem of interest is to model the operational dynamics of the status of each single machine. This model is then integrated to the whole assembly line described above to analyze the whole system. For a single manufacturing machine, there are two underlying processes involve. The first process, the degradation process, in most of the automated assembly systems manifests as long time-scale, fairly regular process (occurring as a result of repeated, "cyclic" loading), as well as short time-scale degradations that occur in components that have steep degradation and restoration rates (short degradation and restoration cycles, such as human attention span, and component misfeeds). The second process, the restoration process, can also be decomposed into a deterministic trend of regular check up or maintenance and random process involving factors such as irregular fixing when random breakdown occurs. We note that the deterministic breakdown and maintenance often take much longer TBF and TTR than the random ones. 
3. Deterministic model for coupled dynamics between degradation and restoration processes of a manufacturing machine

\subsection{Model description}
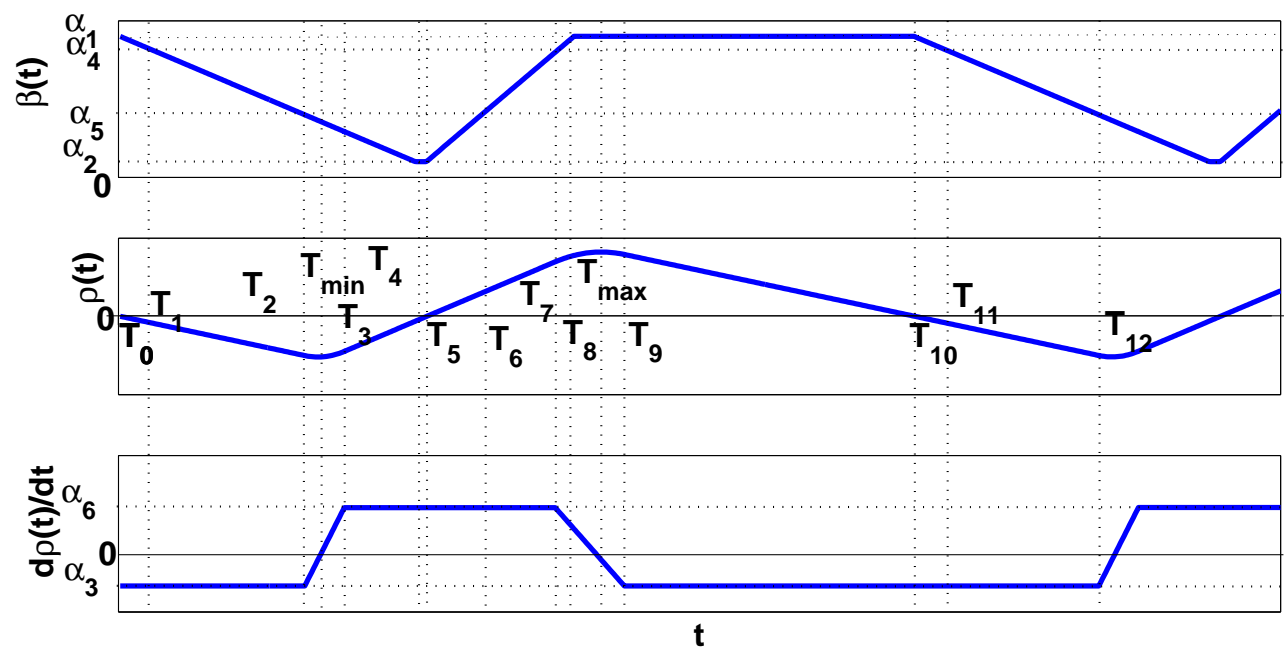

(a)

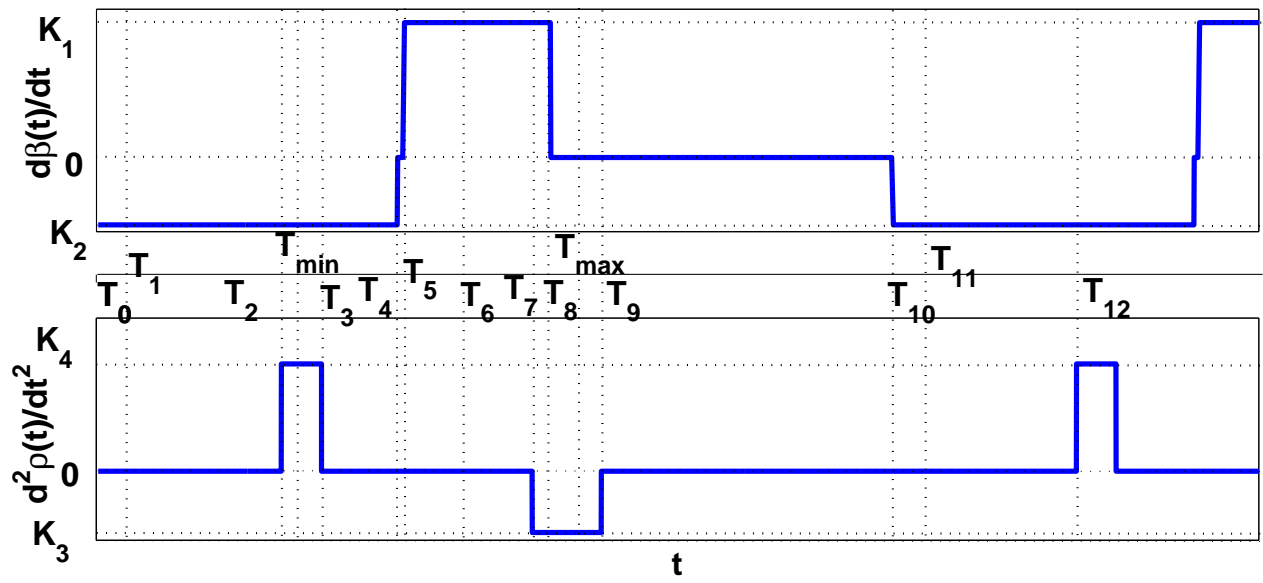

(b)

Figure 1: Illustrative diagram of degradation and restoration variable dynamics

To build the model for a single machine, we look at a sample path as shown in Fig. 1, which captures a representative long times-cale degradation-repair cycles of a manufacturing machine. Here, the restoration rate $\dot{\rho}$ to improve the machine fluctuates around a "nominal" baseline which specifies an average effort (e.g., workforce size) employed for restoration. For simplicity of manipulation and without loss of generality, we choose that "nominal" baseline for $\dot{\rho}$ to be 0 and adjust cumulative restoration $\rho$ as a fluctuation about the linear trend determined by 
this average. The physical connotation of negative $\rho$ and $\dot{\rho}$ is that their values before adjustment are below the "nominal" baseline linear trend and that set level. The downtime due to machine breakdowns is simulated as resulting from a dynamic interplay between the machine degradation condition $\beta$ and the cumulative restoration. We note that a degradation process is often attributed to certain natural laws that have been commonly modeled as piecewise constant rate processes [52]. Real-world repair processes tend to be governed by several humanmachine-interaction considerations [25-29]. For instance, as the condition starts to deteriorate, some attention and resources are expended towards maintaining and repairing the machine. The efforts are significantly ramped up as the machine condition deteriorates further and are phased out as the machine is restored (see Fig. 1). Specifically, upon restoration of machine condition to $\beta(t)=\alpha_{1}$ at $t=T_{0}$, where the machine condition is considered to have reached its peak level, the machine gradually degrades at a constant rate of $K_{2}$. As the condition degrades to $\beta(t)=\alpha_{5}$ at $t=T_{2}$, the restoration rate increases. When $t<T_{4}$, the restoration is not enough to overcome the natural degradation effects, therefore the machine condition continues to decrease and bottoms out to $\beta(t)=\alpha_{2}$. Thereafter, the machine condition starts to improve gradually at a rate of $K_{1}$ at $t=T_{5}$. The restoration rate reaches the maximum level $\dot{\rho}(t)=\alpha_{6}$ at $t=T_{3}$ and retains this value until the machine condition improves to $\alpha_{4}$ at $t=T_{7}$. At $t=T_{7}$, the restoration rate is reduced at a rate of $K_{3}$ until the restoration rate reaches its lowest level of $a_{3}<0$ at $t=T_{9}$. As the restoration rates are phased out at $t=T_{7}$, at first the machine condition continues to increase to reach its peak level at $t=T_{8}$ and retains that value till $t=T_{10}$ when degradation sets in to begin the next such cycle. This dynamic interplay can be captured in its simplest form using the following piecewise constant (nonlinear) differential equations of the form:

$$
\begin{aligned}
\frac{d \beta}{d t} & =K_{1} H_{-}\left(\alpha_{1}-\beta\right) H_{+}(\rho)+K_{2} H_{+}(-\rho) H_{-}\left(\beta-\alpha_{2}\right) \\
\frac{d^{2} \rho}{d t^{2}} & =K_{3} H_{-}\left(\dot{\rho}-\alpha_{3}\right) H_{+}\left(\beta-\alpha_{4}\right)+K_{4} H_{+}\left(\alpha_{5}-\beta\right) H_{-}\left(\alpha_{6}-\dot{\rho}\right)
\end{aligned}
$$

where $\alpha_{1-6}$ are the threshold values of the respective state variables that switch between different regimes, $K_{1-4}$ denote the rates of degradation and restoration- as stated in the foregoing, and $H_{-,+}($.$) represent the switching$ dynamics, which are defined as follows:

$$
H_{-}(x)=\left\{\begin{array}{cc}
0 & x \leq 0 \\
1 & x>0
\end{array}, \quad H_{+}(x)=\left\{\begin{array}{cc}
0 & x<0 \\
1 & x \geq 0
\end{array}\right.\right.
$$


It may be noted that parameters such as $K_{1}, K_{2}, \alpha_{1,2}$ have a specific physical connotation in terms of defining the degradation and restoration rates; the peak and breakdown (degraded) conditions, which may be estimated from shop floor PFS data; and the specifications of a machine operating range. For example, $K_{2}$ is synonymous with the average rate of a specific (here, the long time-scale) degradation mode. It may also be noted that higher order differential equations with more complex forms of non-linearity may be used if the present model structures are found to be inadequate to capture the underlying dynamics. Pertinently, the foregoing affine differential equations $(1,2)$ admit a periodic solution as stated in the following theorem. That periodic solution can capture a recurring, major (large time-scale) mode of breakdown-restoration cycles of real-world manufacturing machine operations.

Theorem 1. If the following conditions hold:

$$
\begin{array}{r}
K_{4}\left(\alpha_{5}-\alpha_{2}\right)+K_{2}\left(\alpha_{6}-\alpha_{3}\right) \geq 0, \\
\alpha_{3}\left(\alpha_{1}-\alpha_{5}\right)+\alpha_{6}\left(\alpha_{5}-\alpha_{2}\right) \leq 0, \\
K_{3}\left(\alpha_{1}-\alpha_{4}\right)+K_{1}\left(\alpha_{6}-\alpha_{3}\right) \geq 0, \\
2 K_{3} \alpha_{6}\left(\alpha_{2}-\alpha_{4}\right)+K_{1}\left(\alpha_{6}^{2}-\alpha_{3}^{2}\right) \geq 0, \\
2 K_{1}\left(-\alpha_{1}+\alpha_{2}\right) \alpha_{6}-K_{3}\left(\alpha_{1}-\alpha_{4}\right)^{2}<0, \\
2 K_{4} K_{1}\left(\alpha_{1}-\alpha_{5}\right)+K_{2}\left(\alpha_{6}^{2}-\alpha_{3}^{2}\right) \geq 0, \\
\alpha_{3}<0<\alpha_{2}<\alpha_{6}<\alpha_{5}<\alpha_{4}<\alpha_{1}, \\
K_{1}>K_{4}>0>K_{2}>K_{3}, \alpha_{3}=-K_{1}
\end{array}
$$

then the set of affine equations $(1,2)$ allows a periodic solution denoted by $x^{*}(t)$ composed of $I=10$ segments, with the period given by

$$
\begin{aligned}
T & =\left(\alpha_{6}-\alpha_{3}\right)\left(\frac{\alpha_{6}-\alpha_{3}}{2 K_{4} \alpha_{6}}+\frac{\alpha_{6}-\alpha_{3}}{2\left(-K_{3}\right)\left(-\alpha_{3}\right)}\right) \\
& +\left(\alpha_{6}-\alpha_{3}\right)\left(\frac{\alpha_{1}-\alpha_{5}}{\alpha_{6}\left(-K_{2}\right)}+\frac{\alpha_{4}-\alpha_{2}}{K_{1}\left(-\alpha_{3}\right)}\right)
\end{aligned}
$$

Proof. See Appendix A.

Remark 1. All the constraints are applied to guarantee the solution follows the desired path, which is popular in practical manufacturing machine. 
The constraints 3(a-d) may be interpreted as follows: Eqn. (3a) reduces to $\frac{\left(\alpha_{6}-\alpha_{3}\right)}{K_{4}} \leq \frac{\left(\alpha_{5}-\alpha_{2}\right)}{-K_{2}}$. This means that the time it takes restoration rate- $\dot{\rho}$ to reach its maximum value must be smaller than the time for the system state $\beta$ to degrade from its peak to the lowest state. Similarly, Eqn. (3c) implies that the time it takes $\dot{\rho}$ to relax to its lowest value from its peak value should be longer than the time for the system state $\beta$ to reach the maximum state from the restoration relaxing state.

From (3b) it follows that the state at which the acceleration of the cumulative restoration- $\rho(t)$ should be initiated is bounded as: $\alpha_{5} \leq \frac{\alpha_{6} \alpha_{2}-\alpha_{3} \alpha_{1}}{\alpha_{6}-\alpha_{3}}$, from (3d) it follows that the state at which the restoration should be reduced is bounded as $\alpha_{4} \geq \frac{K_{1}\left(\alpha_{6}^{2}-\alpha_{3}^{2}\right)}{2\left(-K_{3}\right) \alpha_{6}}+\alpha_{2}$, and Eqn. (3h) defines the highest rate for relaxing the restoration rate $\dot{\rho}(t)$ in terms of the highest effective restoration rate $\dot{\beta}(t)$.

Theorem 2. When the antecedents of Theorem 1 hold, time to failure (TTF), TTR, and availability (A) are given by the following expressions:

$$
\begin{gathered}
T T F=\frac{\alpha_{2}-\alpha_{1}}{K_{2}}+\left(1-\frac{\alpha_{6}}{\alpha_{3}}\right)\left(\frac{\alpha_{4}-\alpha_{2}}{K_{1}}-\frac{\alpha_{6}-\alpha_{3}}{2 K_{3}}\right) \\
T T R=\frac{\left(\alpha_{6}-\alpha_{3}\right)^{2}}{2 K_{4} \alpha_{6}}+\frac{\alpha_{5}-\alpha_{2}}{K_{2}}+\frac{\alpha_{3}\left(\alpha_{1}-\alpha_{5}\right)}{K_{2} \alpha_{6}} \\
\mathbf{A}=\left[K _ { 4 } \left(K_{1}\left(2 K_{3}\left(\alpha_{2}-\alpha_{1}\right) \alpha_{3}+K_{2}\left(\alpha_{3}-\alpha_{6}\right)^{2}\right)\right.\right. \\
\left.\left.-2 K_{2} K_{3}\left(\alpha_{2}-\alpha_{4}\right)\left(\alpha_{3}-\alpha_{6}\right)\right) \alpha_{6}\right] \\
{\left[K_{1}^{3} K_{2} K_{3} \alpha_{3}-2 K_{2} K_{3} K_{4}\left(\alpha_{2}-\alpha_{4}\right)\left(\alpha_{3}-\alpha_{6}\right) \alpha_{6}\right.} \\
+2 K_{1}^{2} K_{3} \alpha_{3}\left(K_{4}\left(\alpha_{5}-\alpha_{1}\right)+K_{2} \alpha_{6}\right) \\
+K_{1} \alpha_{6}\left(K_{2} K_{4}\left(\alpha_{3}-\alpha_{6}\right)^{2}\right. \\
\left.\left.+K_{3} \alpha_{3}\left(2 K_{4}\left(\alpha_{5}-\alpha_{1}\right)+K_{2} \alpha_{6}\right)\right)\right]^{-1} .
\end{gathered}
$$

Proof. See Appendix B.

Remark 2. The following interpretations may be forwarded on how the model parameters influence machine performance characteristics: From (6) it follows that TTR will decrease if $-\alpha_{3}$, the limiting value of $\dot{\rho}$, decreases (which causes $\frac{\left(\alpha_{6}-\alpha_{3}\right)^{2}}{2 K_{4} \alpha_{6}}$ and $\frac{-\alpha_{3}\left(\alpha_{1}-\alpha_{5}\right)}{-K_{2} \alpha_{6}}$ to decrease), or $\alpha_{5}-\alpha_{2}$ increases (which causes $\frac{\alpha_{5}-\alpha_{2}}{-K_{2}}$ to increase). Note that $\alpha_{5}-\alpha_{2}$ increases if $\alpha_{5}$ increases (i.e, we start the restoration process sooner relative to the most degraded state $\alpha_{2}$ ) 
or $\alpha_{2}$ decreases (i.e., the machine has a very low degraded state).

From (5) it follows that TTF will increase if any of the following conditions hold: (i) the range of operating states of a machine $\alpha_{1}-\alpha_{2}$ increases or the effective degradation rate $-K_{2}$ decreases; (ii) the state of the machine at which the restoration rate reduces, $\alpha_{4}$ increases or the maximum effective restoration rate, $K_{1}$, decreases; (iii) the range of restoration rate $\alpha_{6}-\alpha_{3}$ increases, and the maximum restoration rate $\alpha_{6}$ and the peak restoration reducing rate $-\alpha_{3}$ increases. Note that the last two situations imply that $\left(\frac{\alpha_{6}}{-\alpha_{3}}+1\right)\left(\frac{\alpha_{4}-\alpha_{2}}{K_{1}}+\frac{\alpha_{6}-\alpha_{3}}{2\left(-K_{3}\right)}\right)$ increases.

The availability, $\mathbf{A}$, increases if TTR does not increase as TTF increases. For example, when $-K_{3}$, the restoration acceleration decreases (with other parameters unchanged), and TTF increases, with TTR remaining unchanged. This leads to an increase in $\mathbf{A}$.

\subsection{Stability characteristic}

This section is devoted to studying the dynamics behavior including basin of attraction and the stability condition of the solution [61]. Here, the stability of the periodic orbits of such piecewise affine models is defined in terms of the eigenvalues of the Jacobian matrix of a Poincare's map, referred to as a trajectory sensitivity matrix [62]. Stability, at least local, of the periodic solutions is necessary to ensure that the numerical solutions converge over time, at a well-defined mixing rate, with the theoretical steady state periodic orbits. We also provide an expression for the basin of attraction, which can help quantify the robustness of the periodic solution to finite perturbations. The larger the basin of attraction, the more robust are the steady simulation outputs to numerical imprecision and other perturbations. To simplify the model and put it into the general setting of the control theory, $(1,2)$ can be written in the following piecewise affine form

$$
\dot{x}=A_{i} x+B_{i}
$$

where $x \triangleq(\beta, \rho, \dot{\rho})^{T}, A_{i}=A=\left(a_{m n}\right)_{3 \times 3}, i \in\{1, \ldots, 10\}$ such that

$$
a_{m n}= \begin{cases}1 & \text { if }(m, n)=(2,3) \\ 0 & \text { otherwise }\end{cases}
$$


and $B_{i}=\left(b_{1 i}, 0, b_{3 i}\right)^{T}$, with $b_{1 i} \in\left\{K_{1}, K_{2}, K_{1}+K_{2}, 0\right\}, b_{3 i} \in\left\{K_{3}, K_{4}, K_{3}+K_{4}, 0\right\}$. The switching surfaces are 2-dimensional hyperplanes of the form $\mathcal{S}_{i}=\left\{x \mid C_{i} x=d_{i}\right\}, i \in\{1, \ldots, 10\}$ with $C_{i}$ 's given by

$$
\begin{gathered}
C_{1}=C_{2}=C_{4}=C_{6}=C_{7}=C_{8}=\left(\begin{array}{lll}
1 & 0 & 0
\end{array}\right), \\
C_{3}=C_{9}=\left(\begin{array}{lll}
0 & 0 & 1
\end{array}\right), C_{5}=C_{10}=\left(\begin{array}{lll}
0 & 1 & 0
\end{array}\right) .
\end{gathered}
$$

The switching dynamics of the periodic solution (dotted line) stated in Theorem 1 is depicted in Fig. 2a. Let
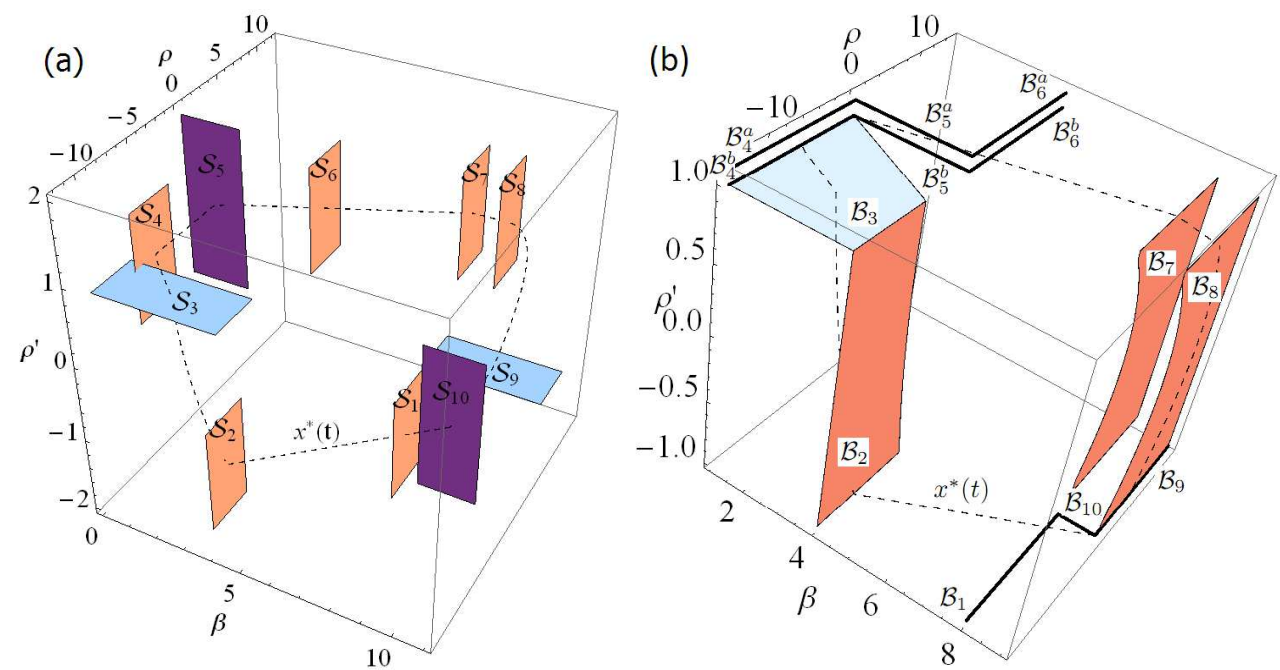

Figure 2: (a) Switching dynamics of a periodic solution (b) Basin of attraction of a periodic trajectory.

us denote by $x_{i}^{*}(t), i=1 . .10$, the segments of a periodic trajectory that starts at $x_{i}^{*}\left(T_{i}\right) \in \mathcal{S}_{i}$ and ends at $x_{i+1}^{*}\left(T_{i+1}\right) \in \mathcal{S}_{i+1}, i=1 . .9$. Let us denote by $\Lambda$ the Poincare map of points in a small neighborhood $\mathscr{B}\left(x_{1}^{*}\right)$ of $x_{1}^{*}\left(T_{1}\right)$ in $\mathcal{S}_{1}$, i.e., $\Lambda: \mathscr{B}\left(x_{1}^{*}\right) \rightarrow \mathcal{S}_{1}$. For such a class of hybrid systems, the Lyapunov theory or Poincare maps [63] are the chief means to investigate stability. The following sufficient condition for the local stability of the periodic solution (limit cycle) follows from this construct.

Proposition 1. [62] The Jacobian $W$ of the Poincare map $\Lambda$ is given by $W=W_{10} W_{9} . . W_{2} W_{1}$, where

$$
W_{i}=\left(E-\frac{v_{i} C_{i+1}}{C_{i+1} v_{i}}\right) e^{A_{i} t_{i}^{*}}
$$

with $v_{i}=A_{i} x_{i+1}^{*}+B_{i}, i \in\{1,2,3, \ldots, 10\}$ and $E$ is the identity matrix. The periodic solution of the system is (a) locally stable if all eigenvalues $\lambda_{j}^{w}$ of $W$ lie strictly within the unit disk, i.e., $\left|\lambda_{j}^{w}\right|<1, j=1 . .3$, and (b) unstable if at least one of the eigenvalues of $W$ lies outside the unit disk. 
Here $W$ is referred to as the trajectory sensitivity matrix. Proposition 1 leads to the following result for the stability of periodic solution $x^{*}(t)$ of $(8)$.

Theorem 3. The periodic solution $x^{*}(t)$ of $(8)$ is locally stable.

Proof. See Appendix C.

The basin of attraction of the locally stable periodic solutions emerging from an initial condition $x^{*}(0)=\left(\beta_{0}, \rho_{0}, \dot{\rho}_{0}\right)$ may be gathered from the following sufficient condition.

Theorem 4. The solution of (1,2) will converge to the periodic solution $x^{*}(t)$ given in Theorem 1 if

$$
\begin{gathered}
\alpha_{5} \leq \beta_{0} \leq \alpha_{4}, \rho_{0} \leq 0, \alpha_{3} \leq \dot{\rho}_{0}<0 \\
K_{2}\left(-2 \rho_{0} K_{4}+\left(\dot{\rho}_{0}-\alpha_{6}\right)^{2}\right)+ \\
2 K_{4}\left(\beta_{0} \dot{\rho}_{0}-\alpha_{2} \alpha_{6}+\alpha_{5}\left(-\dot{\rho}_{0}+\alpha_{6}\right)\right) \leq 0
\end{gathered}
$$

and the inequalities specified in (3) hold for the model parameters.

Proof. See Appendix D.

From the proof (Appendix D), the constraints specified in (11) ensure that the system evolution patterns shadow the periodic solution $x^{*}(t)$; specifically, the system state converges to (actually merges with) $x_{5}^{*}\left(T_{5}\right)$, the initial state of the $5^{\text {th }}$ segment on the trajectory of $x^{*}(t)$.

Remark 3. Eqn. (11) implies that initial degradation state $\beta_{0}$ is bounded between starting restoration- state $\alpha_{5}$ and relaxing restoration state $\alpha_{4}$; the initial cumulative restoration $\rho_{0}$ and the restoration rate $\dot{\rho}_{0}$ are negative, and $\rho_{0}$ and $\dot{\rho}_{0}$ must fulfill the quadratic constraint (11b).

The basin of attraction may also be derived from combining basins of attraction on all switching surfaces[62]. The basin of attraction (composed of the 4 shaded regions and the bold lines) specified in the following Theorem are depicted in Fig. 2b.

Theorem 5. The trajectories that start from the following specified regions on the switching surfaces converge to the limit cycle $x^{*}(t)$ defined in Theorem 1

$$
\text { On } \mathcal{S}_{1}: \dot{\rho}_{0}=\alpha_{3}, \rho_{0} \leq \max \left\{0, \frac{\left(\alpha_{3}\left(\alpha_{4}-\alpha_{5}\right)+\left(-\alpha_{2}+\alpha_{5}\right) \alpha_{6}\right)}{K_{2}}+\frac{\left(\alpha_{3}-\alpha_{6}\right)^{2}}{2 K_{4}}\right\}
$$


On $\mathcal{S}_{2}: \max \left\{-\frac{K_{4}\left(\alpha_{2}-\alpha_{5}\right)}{K_{2}}+\alpha_{6}, \alpha_{3}\right\} \leq \dot{\rho}_{0}<\alpha_{6}, \dot{\rho}_{0}^{2} \geq \alpha_{6}^{2}+2 K_{4} \rho_{0}, \rho_{0} \leq 0,2 K_{4}\left(\alpha_{2}-\alpha_{5}\right) \alpha_{6}>K_{2}\left(\left(\dot{\rho}_{0}-\alpha_{6}\right)^{2}-2 K_{4} \rho_{0}\right)$.

On $\mathcal{S}_{3}: \alpha_{2}<\beta_{0} \leq \alpha_{5}, \alpha_{6}\left(-\alpha_{2}+\beta_{0}\right)<K_{2} \rho_{0}$.

On $\mathcal{S}_{4}:\left\{\rho_{0}<0, \dot{\rho}_{0}=\alpha_{6}\right\}$ or $\left\{\rho_{0}<0, \dot{\rho}_{0}=-\frac{K_{3}\left(\alpha_{1}-\alpha_{4}\right)}{K_{1}}+\alpha_{6}\right\}$.

On $\mathcal{S}_{5}:\left\{\dot{\rho}_{0}=\alpha_{6}, \min \left\{\alpha_{5}, \frac{K_{3}\left(\alpha_{1}-\alpha_{4}\right)^{2}}{2 K_{1} \alpha_{6}}+\alpha_{1}, \frac{K_{1}\left(\alpha_{3}^{2}-\alpha_{6}^{2}\right)}{2 K_{3} \alpha_{6}}+\alpha_{4}\right\} \geq \beta_{0} \geq \alpha_{2}\right\}$ or $\left\{\dot{\rho}_{0}=-\frac{K_{3}\left(\alpha_{1}-\alpha_{4}\right)}{K_{1}}+\alpha_{6}\right.$,

$\left.\min \left\{\alpha_{5}, \frac{K_{3}\left(\alpha_{1}-\alpha_{4}\right)^{2}}{2 K_{1} \alpha_{6}}+\alpha_{1}, \frac{K_{1}\left(\alpha_{3}^{2}-\alpha_{6}^{2}\right)}{2 K_{3} \alpha_{6}}+\alpha_{4}\right\} \geq \beta_{0} \geq \alpha_{2}\right\}$.

On $\mathcal{S}_{6}:\left\{\dot{\rho}_{0} \geq \frac{-K_{3}\left(\alpha_{1}-\alpha_{4}\right)}{K_{1}}+\alpha_{6}, \rho_{0} \geq 0, K_{3}\left(\alpha_{1}-\alpha_{4}\right)^{2}+2 \dot{\rho}_{0} K_{1}\left(\alpha_{1}-\alpha_{5}\right)+2 K_{1}^{2} \rho_{0}>0\right.$,

$\left.2 \dot{\rho}_{0} K_{3}\left(\alpha_{4}-\alpha_{5}\right)+K_{1}\left(-\dot{\rho}_{0}^{2}+\alpha_{3}^{2}+2 K_{3} \rho_{0}\right)<0\right\}$.

On $\mathcal{S}_{7}:\left\{\rho_{0} \geq 0, \dot{\rho}_{0}=\alpha_{6}\right\}$ or $\left\{\rho_{0} \geq 0, \dot{\rho}_{0}=-\frac{K_{3}\left(\alpha_{1}-\alpha_{4}\right)}{K_{1}}+\alpha_{6}\right\}$.

On $\mathcal{S}_{8}:\left\{\rho_{0}>0, \alpha_{3}<\dot{\rho}_{0} \leq \alpha_{6}, \alpha_{3}^{2}+2 K_{3} \rho_{0}<\dot{\rho}_{0}^{2}\right\}$.

On $\mathcal{S}_{9}:\left\{\beta_{0}=\alpha_{1}, \rho_{0}>0\right\}$.

On $\mathcal{S}_{10}:\left\{\rho_{0}>0, \dot{\rho}_{0}=\alpha_{3}, \alpha_{1} \geq \beta_{0}>\max \left\{\alpha_{4},-\frac{K_{2}}{2 K_{4} \alpha_{3}}\left(\alpha_{3}-\alpha_{6}\right)^{2}+\frac{1}{\alpha_{3}}\left(\alpha_{2} \alpha_{6}+\alpha_{5}\left(\alpha_{3}-\alpha_{6}\right)\right), \frac{K_{2}\left(-\alpha_{3}{ }^{2}+\alpha_{6}^{2}\right)+2 \alpha_{3} K_{4} \alpha_{5}}{2 \alpha_{3} K_{4}}\right\}\right\}$.

Proof. See Appendix E.

Remark 4. Note that all the constraints used to bound the basin of attraction on each switching surface are added to sufficiently guarantee that the switching order of the periodic solution of Eqs. (1,2) is maintained. As a consequence, the system returns to a point on $x^{*}(t)$. Thus, the system is stable.

Remark 5. The basin of attraction formulated using the earlier method in [62] is constructed in 2 steps. The first step bounds a set in form of ellipsoids from which the impact maps [62] are contracted. This set is then shrunk to maintain the switching order. For the present system, some impact maps are not contracting. This leads to the following result.

Proposition 2. The basin of attraction formulated in Theorem 5 contains the basin of attraction derived using the method in [62].

This result is part of the proof represented in Appendix E.

\section{Stochastic model for modeling short time breakdown and repair of a single machine and the multimodal distribution}

In this Section, we will extend the model in $(1,2)$ to include the effects of minor breakdowns that happen in short time scale by introducing a stochastic form of $\alpha_{2}$ and $K_{2}$. As shown in Fig. 6b, data collected in this study exhibits 
a multimodal distribution. The right mode presents major breakdowns that happen in long time-scale and can be modeled by the deterministic model in Section 3. The left mode presents minor breakdowns happen randomly in short time scale and will be considered in this Section.-For the following Theorem, we consider the system in one period, i.e., $0 \leq t \leq T$.

Theorem 6. If antecedents of Theorem 1 hold and $\alpha_{2}$ and $K_{2}$ are as follows:

$$
\begin{aligned}
& \alpha_{2}(t)= \begin{cases}\alpha_{2}^{0}-\gamma(t)+\int_{T_{4}}^{t} K_{2}^{0} H_{+}(\gamma(\tau)) d \tau & \text { if } T_{4} \leq t<T_{5}-\epsilon \\
\alpha_{2}^{0}-\varepsilon \tilde{K}_{2} & \text { if } T_{5}-\epsilon \leq t \leq T_{5} \\
\alpha_{2}^{0} & \text { otherwise }\end{cases} \\
& \tilde{K}_{2}=-\frac{1}{\varepsilon} \int_{T_{4}}^{T_{5}-\epsilon} K_{2}^{0} H_{+}(\gamma(\tau)) d \tau . \\
& K_{2}(t)= \begin{cases}\tilde{K}_{2} & \text { if } T_{5}-\varepsilon \leq t<T_{5} \\
K_{2}^{0} & \text { otherwise }\end{cases}
\end{aligned}
$$

where $0<\varepsilon<T_{5}-T_{4}$, then TBF and TTR in this period exhibit right skewed distributions with skewness in the range of 1.4-2. Here, $\gamma(t)$ is a random process whose time intervals between successive zero-crossings follow exponential distribution with rate $\nu ; \alpha_{2}^{0}, K_{2}^{0}$ are the values, respectively, of $\alpha_{2}$, and $K_{2}$ in the deterministic model $(1,2) ;$ and $T_{4}=\left(\frac{\alpha_{1}-\alpha_{2}}{-K_{2}}\right), T_{5}=\frac{\left(K_{1}+\alpha_{6}\right)\left(2 K_{4}\left(\alpha_{5}-\alpha_{1}\right)+K_{2}\left(K_{1}+\alpha_{6}\right)\right)}{2 K_{2} K_{4} \alpha_{6}}$.

Proof. See Appendix F for the proof and a sample path of the solution in this case.

Note that in this case, TTR is the time interval between a zero up-crossing and the next zero down-crossing, and TBF is the time interval between 2 consecutive zero down crossings of $\beta(t)-\alpha_{2}(t)$. The following result shows that our model can capture the multimodal characteristic of TBF.

Corollary 1. Model (1,2) with $\alpha_{2}, K_{2}$ as proposed in (12,14) when $t \leq T$ and $\alpha_{2}(t)=\alpha_{2}(t \bmod T)$ when $t \geq T$ can generate TBF that exhibit a multimodal distribution.

Proof. See Appendix G. 


\section{Numerical validation}

In this section, we report 2 case studies. The data sets employed in these case studies contain TBFs and TTRs collected from manufacturing machines of an assembly line of a leading automobile manufacturer. The first study assesses how well the model parameters estimated based on the foregoing theoretical results can capture the distribution of TBF and TTR of each machine in an assembly line. The second case study evaluates the extent to which the model, with parameters "optimally" estimated using a genetic algorithm [64], can be used to simulate the dynamics of the whole assembly line.

\subsection{Performance of the model when applying to a single machine}

First, we investigated the distributions of TBF from real world data over short time scales, i.e, TBF $<10$ min. As shown in Fig. 3a, the data exhibit a highly right skewed distribution over the short time-scales. The estimated parameters $\left(\hat{\nu}_{l}\right)$ of the exponential distribution models of the short time-scale TBF distribution of various manufacturing machines $l=1,2, \ldots, 14$, (see Table 1 ) suggest that for most machines, the $R^{2}$ for the exponential fit is high, around $90 \%$, and the standard deviation is about $10 \%$ of the estimates in many cases. This result implies that $\ln (1-\widehat{F(T B F)})$ trends linearly with TBF values, and short-term TBF and TTR can therefore be approximated using an exponential distribution.

Table 1: Summary of exponential fitting of short-term TBF samples from a real-world manufacturing system.

\begin{tabular}{|c|c|c|c|c|c|c|c|}
\hline $\mathrm{MC}$ & $\hat{\nu}$ & $R^{2}$ & $\sigma(\% \hat{\nu})$ & $\mathrm{MC}$ & $\hat{\nu}$ & $R^{2}$ & $\sigma(\% \hat{\nu})$ \\
\hline 1 & 0.23 & 0.93 & 12.68 & 8 & 0.26 & 0.89 & 17.19 \\
\hline 2 & 0.30 & 0.95 & 9.79 & 9 & 0.32 & 0.83 & 18.86 \\
\hline 3 & 0.28 & 0.97 & 8.24 & 10 & 0.31 & 0.95 & 10.43 \\
\hline 4 & 0.29 & 0.89 & 16.15 & 11 & 0.16 & 0.93 & 18.56 \\
\hline 5 & 0.22 & 0.84 & 24.70 & 12 & 0.34 & 0.88 & 17.03 \\
\hline 6 & 0.30 & 0.88 & 19.34 & 13 & 0.28 & 0.97 & 8.24 \\
\hline 7 & 0.25 & 0.70 & 27.23 & 14 & 0.32 & 0.88 & 15.95 \\
\hline
\end{tabular}

Second, we compared the distributions of short time-scale TBF and TTR from the data vs. those generated from the model for the 14 machines of the assembly line. For each machine, the model $(1,2)$ was parametrized with the value estimated in Table 1, and those values that meet the antecedents of Theorem 3 and the perturbations specified in $(12,13)$ to mimic the degradation dynamics (e.g., $\hat{\nu}=0.276$ for Machine 6 ) and $\varepsilon \approx 0.001$. The model with these parameter settings was used to simulate a 600 min long operation of an assembly segment with step size set at 0.01 min. The simulations were replicated 20 times. The closeness of the distributions of model-derived successive zerocrossing $\left(\alpha_{2}\right.$-crossing) intervals (mimicking TTR) to exponential distribution, $\exp \left(\hat{\nu}_{l}\right)$, for each machine was assessed 
using a Kolmogorov-Smirnov (KS) test. Parenthetically, the KS statistic, $\kappa$, is the maximum difference between empirical cdf $\widetilde{F(T B F)}$ and the cdf of $\exp (\hat{\nu})$. The results suggest that the short time-scale TTR from simulations for Machine $k$ are statistically indistinguishable from an exponential distribution $\exp \left(\hat{\nu}_{k}\right)$ (median $\mathrm{p}$-value of about $0.6)$.

Third, we ran simulations to verify the similarity of overall distributions of simulated vs. actual TBF and TTR. We set the simulation time span to $6000 \mathrm{~min}$ and the step size to $0.001 \mathrm{~min}$. As evident from Fig. 3 and statistical tests, the histograms of model-generated TBF and TTR for a machine hold a distinct similarity to those from real-world data in that (a) both show a very similar right skewed distribution over short times and (b) the second mode occurs in the same vicinity (here, $\mathrm{TBF}=1800 \mathrm{~min}$ ), but the real-world data shows larger dispersion compared to the model-derived distribution.

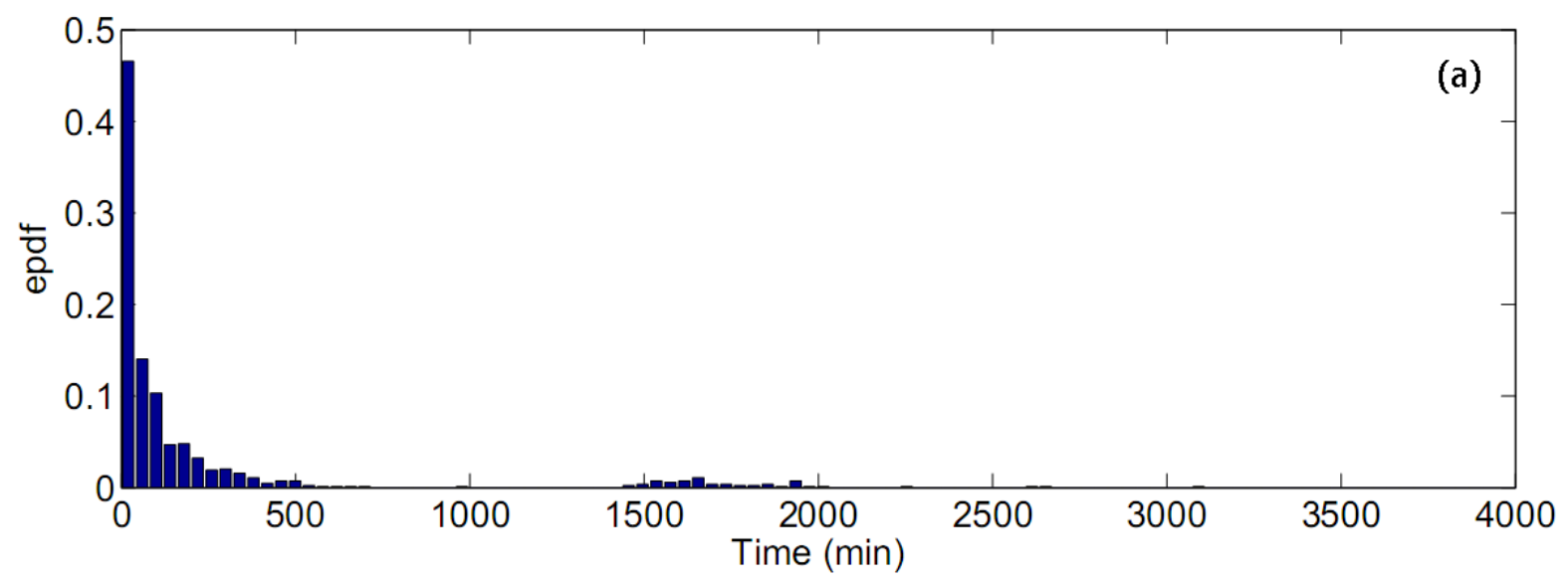

(a)

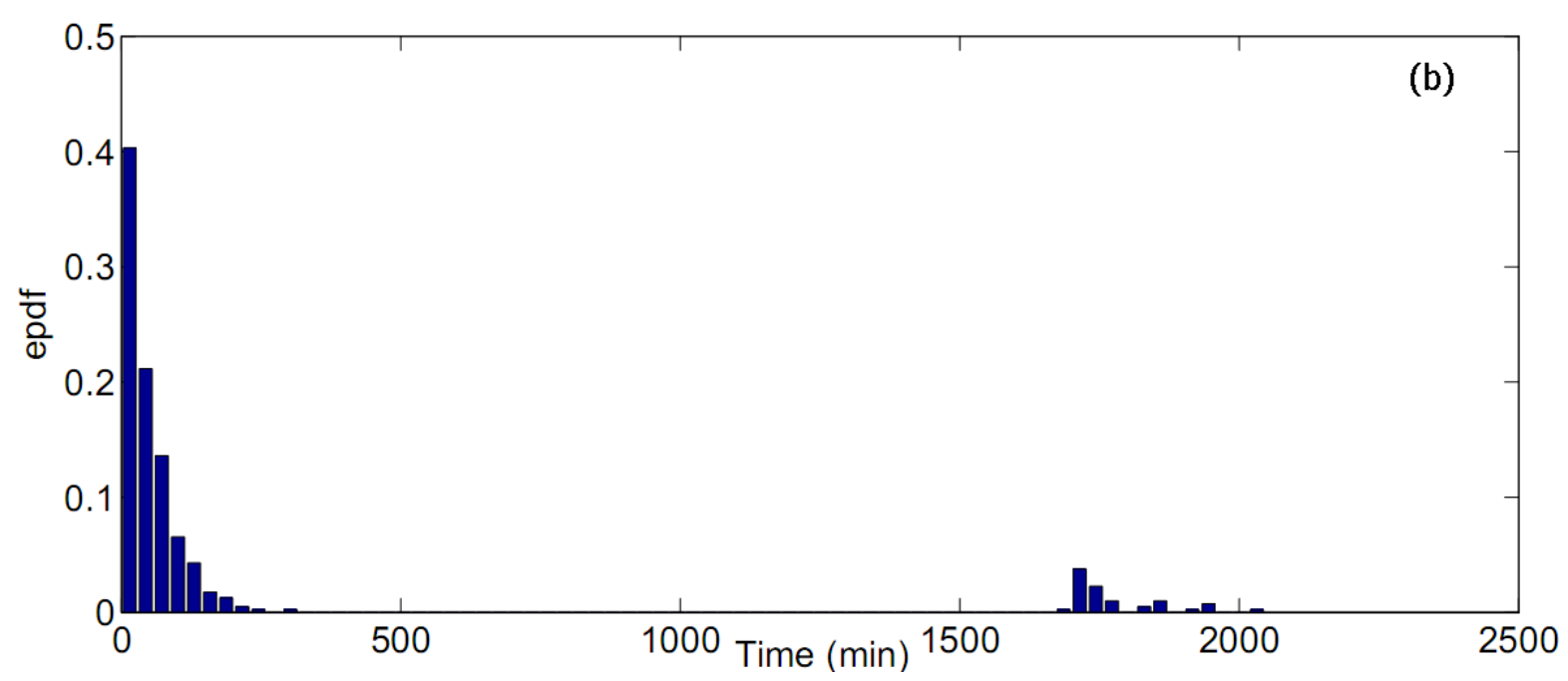

(b)

Figure 3: Comparison of histograms of TBF data from a real-world manufacturing assembly machine (station) (a) with that from simulations (b). 


\subsection{Performance of the model when integrating to whole assembly line}

In this case study, we applied the foregoing modeling approach to capture the dynamics of machine breakdowns as well as their effect on the throughput in an automotive manufacturing assembly line segment.

As mentioned above, flow modeling approaches have traditionally been used for qualitative analysis of system dynamics and not for real-time performance estimation. Discontinuities in flow caused by blocking, starving, and breakdown events adversely affect the performance of integration routines used to solve the model, and often cause the numerical solution procedures to become unstable. To overcome this drawback, the Heaviside function was replaced by sigmoid function theory $[65,66]$. Note that the rationale for this replacement is that sigmoidal functions $\operatorname{sgm}(w x)=\frac{1}{1+e^{-w x}}$ can approximate the Heaviside functions $H_{-,+}(x)$ and improve the efficiency of numerical simulation. It may also be noted that when $w$ gets large enough, we have

$$
\lim _{w \rightarrow \infty} \operatorname{sgm}(w x)=H_{+,-}(x)
$$

Therefore, we can derive approximated vector flow fields that pose fewer numerical instability issues and to facilitate faster simulation of system dynamics. For example, the time taken to generate the solutions shown in Fig. 1 for degradation dynamics decreased 2146 times with the use of sigmoidal functions. By using the sigmoidal function of the form $\operatorname{sgm}(x)=\left(1+e^{-x}\right)^{-1}$, the coupled dynamics of degradation and restoration $(1,2)$ may be rewritten as:

$$
\begin{aligned}
d \beta / d t & =K_{1} \operatorname{sgm}\left(w_{1}\left(\alpha_{1}-\beta\right)\right) \operatorname{sgm}\left(w_{2} \rho\right) \\
& +K_{2} \operatorname{sgm}\left(-w_{3} \rho\right) \operatorname{sgm}\left(w_{4}\left(\beta-\alpha_{2}\right)\right) \\
\left(d^{2} \rho\right) /\left(d t^{2}\right) & =K_{3} \operatorname{sgm}\left(w_{5}\left(\dot{\rho}-\alpha_{3}\right)\right) \operatorname{sgm}\left(w_{6}\left(\beta-\alpha_{4}\right)\right) \\
& +K_{4} \operatorname{sgm}\left(w_{7}\left(\alpha_{5}-\beta\right)\right) \operatorname{sgm}\left(w_{8}\left(\alpha_{6}-\dot{\rho}\right)\right)
\end{aligned}
$$

and the throughput rate is adjusted to account for degradation and starve/block conditions in each machine as

$$
u_{k}(t)=\mu_{k}(t) \operatorname{sgm}\left(L_{k}(t)\right) \operatorname{sgm}\left(L_{k+1}^{\max }-L_{k+1}(t)\right) \operatorname{sgm}\left(\beta_{k}(t)-\beta_{k}^{0}\right)
$$

As noted earlier, parametrization of the model using historical fault realizations, such as historical records of TBF and TTR, can be significantly challenging. Instead of using a feasible set based on theoretical results, we used 
a genetic algorithm (GA) to "optimally" select the n-SDE models for each station (labeled Machine 1 through Machine 18) in the real-world manufacturing assembly line segment. In essence, GA performs a stochastic search to determine the model parameters so that the marginal distribution (histogram) of TBF and TTR estimated from the simulation closely match with those from actual data. Notably, the parameters are tuned to capture only the marginal distributions of TTR and TBF. The structure of the differential equations essentially accounts for the TBF-TTR dependencies. The fitness (or the objective) function, $O p$, of the GA is expressed in terms of the empirical marginal distributions of TTR and TBF as follows

$$
\begin{aligned}
O p & =\omega_{1}\left(\frac{\sum_{j=1}^{N_{0}}(H i s t T B F(j)-H i s t T B F a(j))^{2}}{N_{0}}\right. \\
& \left.+\frac{\sum_{j=1}^{N_{1}}(H i s t T T R(j)-H i s t T T R a(j))^{2}}{N_{1}}\right) \\
& +\omega_{2}\left(\frac{\sum_{j=N_{0}+1}^{N_{0}+N_{2}}(H i s t T B F(j)-H i s t T B F a(j))^{2}}{N_{2}}\right. \\
& \left.+\frac{\sum_{j=N_{1}+1}^{N_{1}+N_{3}}(H i s t T T R(j)-H i s t T T R a(j))^{2}}{N_{3}}\right) \\
& +\omega_{3}\left(\frac{\sum_{j=N_{0}+N_{2}+1}^{N_{0}+N_{2}+N_{4}}(H i s t T B F(j)-H i s t T B F a(j))^{2}}{N_{4}}\right. \\
& \left.+\frac{\sum_{j=N_{1}+N_{3}+1}^{N_{1}+N_{3}+N_{5}}(H i s t T T R(j)-H i s t T T R a(j))^{2}}{N_{5}}\right)
\end{aligned}
$$

Here, the distributions of TBF and TTR estimated from the histogram transformations (HistTBF and HistTTR) of the model solutions were compared with those from the actual fault data (HistTBFa and HistTTRa), and $j$ is the histogram bin index. The histogram bins of TBF from the model (HistTBF) and the actual data (HistTBFa) were partitioned into the same three groups, each with $N_{0}, N_{2}, N_{4}$ bins. The first $N_{0}$ bins capture the nearexponential distribution of the short TBFs, the next $N_{2}$ bins capture the steady non-exponential distribution of the intermediate values of $\mathrm{TBF}$, and the last $N_{4}$ bins capture the second mode and beyond of the (large) TBF distribution. Similarly, $N_{1}, N_{3}, N_{5}$ are the corresponding bins used to compare histogram transformations of TTR distribution from the model (HistTTR) with actual historic data (HistTTRa). The weights for the three areas of the histogram, $\omega_{1}, \omega_{2}, \omega_{3}$, were assigned to be 100,10 , and 1 , respectively, to represent their relative importance from an operational standpoint. The simulated as well as the actual TBF and TTR data were gathered over a two-month period. The decision variables for GA optimization include the structural parameters of the model, namely, $\alpha_{1-6}, K_{1-4}, w_{1,4,5,8}, w_{2}\left(=w_{3}\right)$, and $w_{6}\left(=w_{7}\right)$, and perturbation parameter $\nu$. The GA essentially tuned 


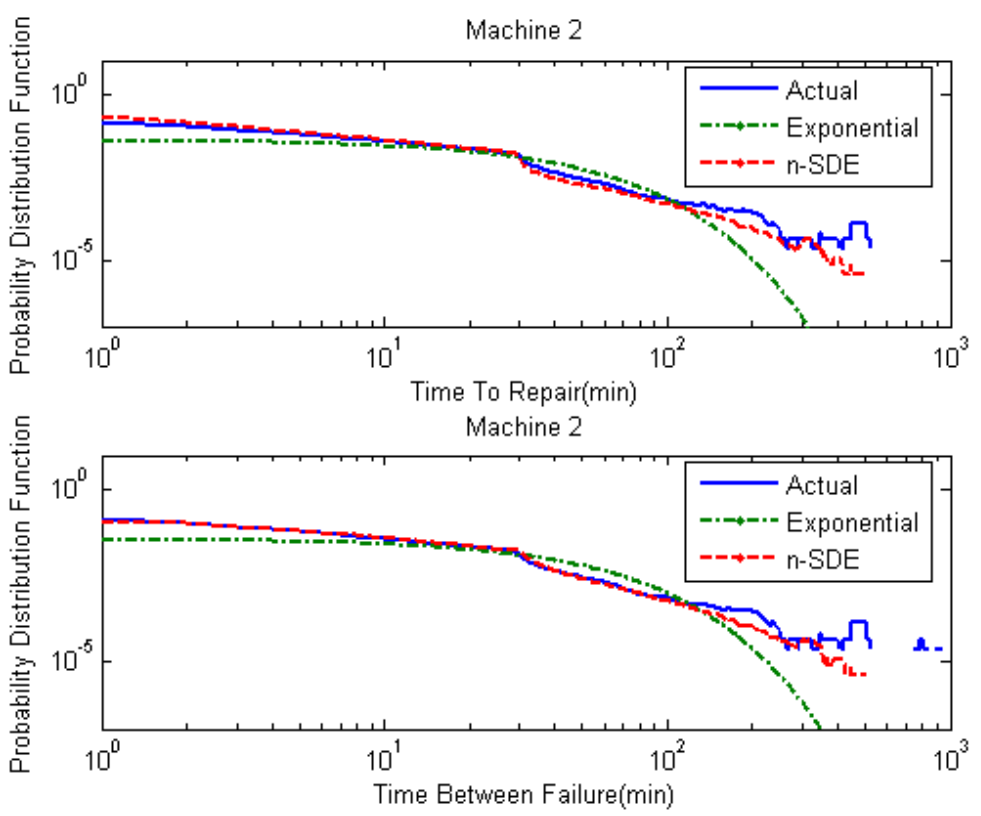

Figure 4: Comparison of marginal pdfs of TTR and TBF obtained from actual data (blue), n-SDE model (red), and exponential distribution model (green) for the representative case of Machine 2

these 17 model parameters to reduce Op below a threshold (stopping criterion). Each parameter was modeled as a 35-bit long string. Stochastic optimization methods, such as GAs are known to locate the basin (trough/crest) of the global optimum with a very high probability [67]. For the cases examined, the GA was found to converge to a near-plateau in at most 50 iterations. A Nelder-Mead simplex (NM) method was used to refine the fitness of the solutions because, once the fitness improvement rate drops below a threshold, it is assumed that the parameter values lie in the basin of the global minimum. The resulting GA-parametrized model appears to adequately capture the dynamics of machine degradation, as well as the distributions of TBF and TTR, better than conventional exponential distribution models parametrized from actual data. For instance, Fig. 4 shows the marginal PDFs of TTR for a representative assembly station (Machine 2) obtained from the actual historical data (solid blue line) vs. the distribution obtained from the model (red dashed line) vs. the one obtained using the best fit exponential distribution (green dashed dotted line).

It may be noted that the pattern observed for Machine 2 is representative of those from most of the other assembly stations barring the ones at either end and the parallel stations. The results suggest that the exponential model underestimates the left tails of TBF and TTR densities by one order of magnitude compared to the actual for all 18 machines. More specifically, the mean square error (MSE) of the distributions from the model is one order in magnitude lower than those from the exponential model. This is likely because the parametrized model can capture the distributions of TBF and TTR much better than the exponential models for short times (i.e., the first minute) 
Table 2: Comparison of mean, standard deviation of TTR and TBF for Machine 2 estimated from n-SDE model vs. actual data

\begin{tabular}{|c|c|c|c|c|c|c|c|}
\hline \multicolumn{2}{|c|}{ Mean TTR } & \multicolumn{2}{c|}{ Std Dev TTR } & \multicolumn{2}{c|}{ Mean TBF } & \multicolumn{2}{c|}{ Std Dev TBF } \\
\hline Actual & Model & Actual & Model & Actual & Model & Actual & Model \\
\hline 9.41 & 9.24 & 13.59 & 15.11 & 27.35 & 24.71 & 29.42 & 25.28 \\
\hline \multicolumn{2}{|c|}{$\%$ dev } & \multicolumn{2}{|c|}{$\%$ dev } & \multicolumn{2}{c|}{$\%$ dev } & \multicolumn{2}{c|}{$\%$ dev } \\
\hline \multicolumn{2}{|c|}{$1.81 \%$} & \multicolumn{2}{|c|}{$10.1 \%$} & \multicolumn{2}{c|}{$9.67 \%$} & \multicolumn{2}{c|}{$14.05 \%$} \\
\hline
\end{tabular}

as shown in Fig. 4, as well as times beyond 100 minutes. Pertinently, Inman's work[68] suggests that exponential distribution may be adequate to capture TTR and TBF distributions in many cases. Consequently, the industry has been using exponential distribution in many of its simulations. The current assembly line segment appears to be an exception to this earlier finding. For example, we noticed significant cross-correlation (0.25-0.55 for large samples) between TTR and TBF for 6 out of 18 machines. TTR and TBF sequences also exhibited significant auto-correlations and/or nonlinear dependencies [19]. The proposed modeling approach may be a suitable means to capture the variations of TTR and TBF more accurately and to model manufacturing systems with more generic failure distributions and coupled dynamics. The improved model parametrization mentioned above leads to fairly close estimation of the mean (i.e., MTBF, MTTR) and standard deviations of the respective distributions, as shown in Table 2 for Machine 2 .

Figs. 5a and 5b provide a comparison of MTBF and MTTR, respectively, for the 18 machines from the model vs. the actual data. The actual statistics are shown in light green bars (lighter shade) and the model outputs are shown in dark brown (darker shade). The results suggest that MTBF computed from the model lies within 3-10\% of that computed from the actual data for 10 of the 18 machines. The model-estimated MTBF values for the remaining 8 machines vary on an average by $24 \%$ relative to those computed from actual data. Such large discrepancies may be caused by the inadequacy of the model structure, despite improvements over a simple exponential distribution model, to capture all the salient features of the real-world TBF data. Similarly, the model-estimated MTTR values for 10 machines vary between $2-10 \%$ of those computed from the actual data, and vary by an average of $28 \%$ from those computed from the actual data for the remaining 8 machines. These results indicate that for 10 out of the 18 machines the parametrized n-SDE model captures the traditional statistical quantifiers TBF and TTR. Interestingly, the exponential model was also found to capture the means, but the errors in capturing the standard deviations were $>50 \%$. Although an average of $24 \%$ and $28 \%$ deviations may appear high in comparison to the close agreement found for the other 10 machines, it may be noted that the traditional statistical metrics alone are not sufficient to capture the entire spectrum of dynamic behaviors; i.e., the effect of some of these deviations may 


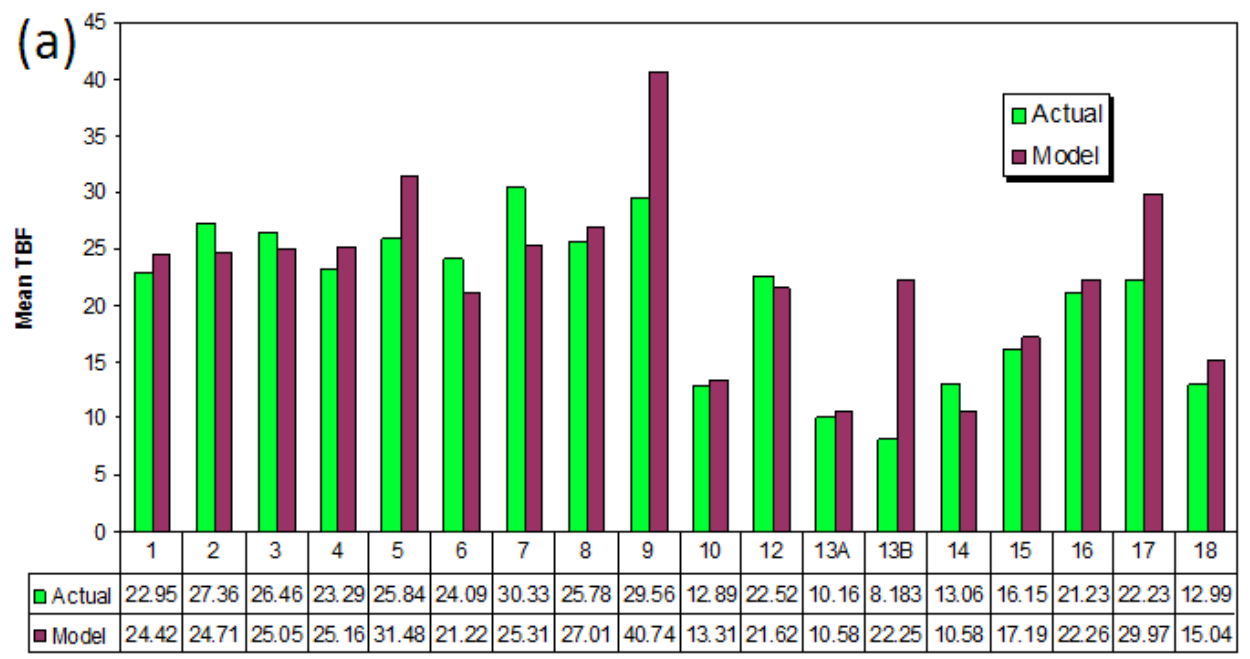

(a)

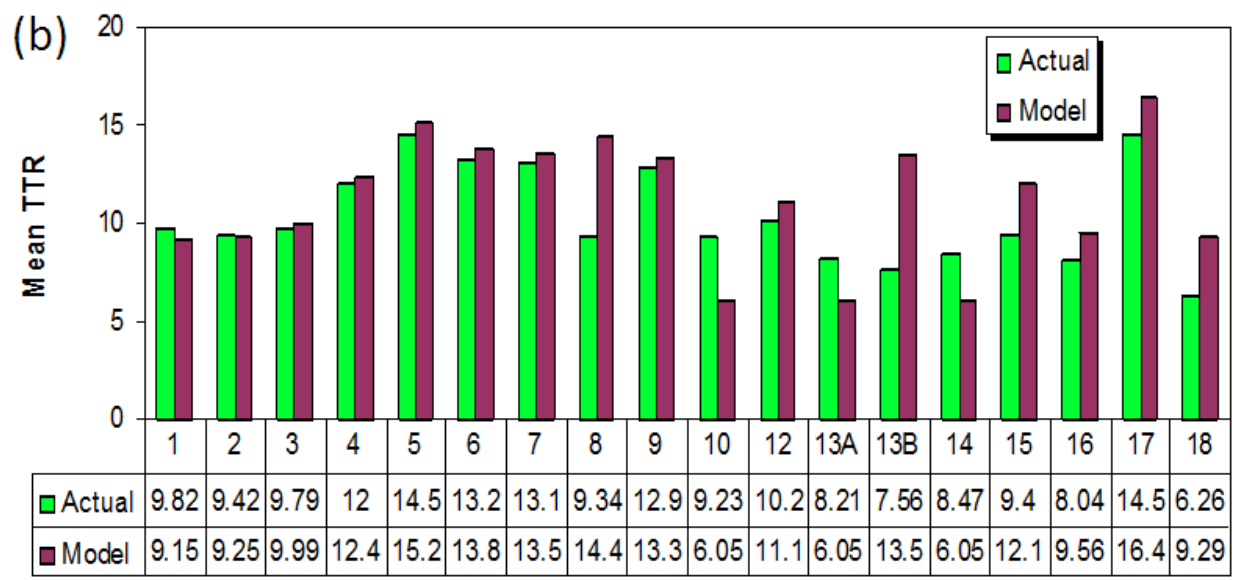

(b)

Figure 5: Comparison of (a) mean TBF and (b) mean TTR from the n-SDE model with those realized for various machines in an actual manufacturing system scenario 

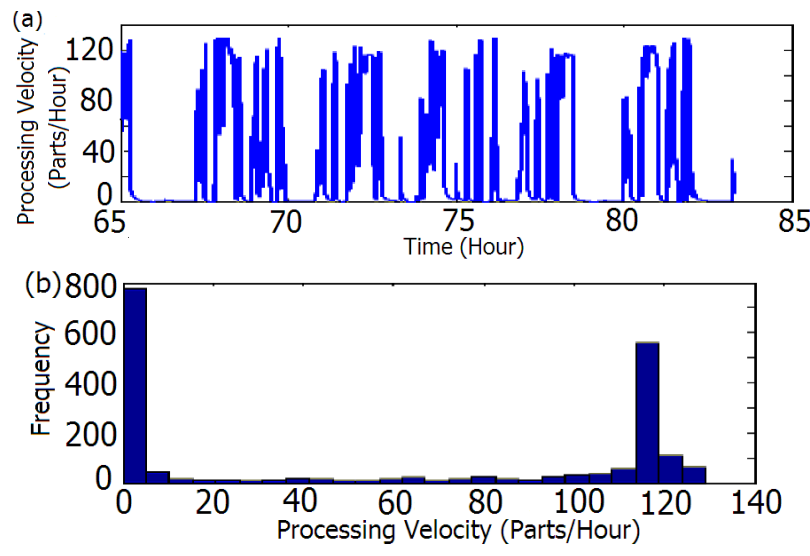

Figure 6: (a) Time-Portrait of processing velocity for Machine 2. (b) Histogram of the processing velocity for Machine 2

not be high in terms of affecting the dynamics of throughputs.

Next, we investigated the extent to which this improvement in capturing the distributions of TTR and TBF translates into capturing the throughputs (or processing velocity in the case of fluid models). Fig. 6a shows the variations of processing velocity with time for Machine 2. It indicates that the model is able to capture the aperiodic nature (see Fig. 6a), and multimodal distributions (see Fig. 6b) of the processing velocities. These observations, shown in the context of Machine 2, are consistent across all the machines in the investigated assembly line segment. The maximum processing velocity or throughput rate for Machine 2 (obtained from the actual data) is $129 \mathrm{jobs} / \mathrm{hr}$. This value is likely to occur, as is evident from Eqn. (15), whenever the upstream buffer is stocked, the downstream buffer is near-empty and the machine is up. Also, the machine was operating near its peak velocity of $129 \mathrm{jobs} / \mathrm{hr}$ for $<2.5 \%$ of the two-month-long observation period and at zero for roughly $28 \%$ of the time. The average jobs/hour (processing velocity) from the actual and the model are 37.29 jobs/hour and 39.50 jobs/hour, respectively. The deviation between the model and the actual is $5.59 \%$, which makes them quite comparable. In contrast, the deviations with DES models that employ exponential TBF and TTR distributions exceeded $20 \%$ for most machines.

We also compared the n-SDE model simulation time with that of a DES model with exponential TBF and TTR distributions. Essentially, there is not much difference in computational effort between DES and n-SDE for simulating short time-scale TBF and TTR. However, for simulating large time-scale TBF and TTR, the fluid flow approximation of parts movement can significantly reduce computation time. Although the coupled dynamics of TTR and TBF considered in the n-SDE model can affect computation time, the simulations are initiated with "good" initial conditions that are in the basin of attraction (i.e., close to the steady state). The total computation 
time was reduced on average by about $25 \%$ with the use of the sigmoidal function per simulation of 100 time units using MATLAB-Simulink for every run over the simplest of DES simulation conducted in an efficient simulation environment (ARENA), with an added advantage of capturing the dependencies and multimodal distributions. Also, many of the simulations happen near the steady state, and the short-time events are separated out from the large time-scale, steady state, near-periodic breakdowns and repairs. Therefore, the almost ergodicity of the process was used to avoid a large number of replications.

Table 3: Comparison of recurrence quantifiers of TBF time-series realized in actual PFS data loggers with that from n-SDE and exponential models, respectively

\begin{tabular}{|c|c|c|c|c|c|}
\hline \multirow{2}{*}{ Metric } & \multicolumn{3}{|c|}{ TTR } & \multicolumn{2}{c|}{ TTR Error \% } \\
\cline { 2 - 6 } & Actual & Model & Exp & Model & Exp \\
\hline Recrate & 39.94 & 24.7 & 0.04 & $38 \%$ & $100 \%$ \\
\hline Determinism & 97.48 & 96.94 & 25.32 & $1 \%$ & $74 \%$ \\
\hline Laminarity & 115 & 82 & 8 & $29 \%$ & $90 \%$ \\
\hline Entropy & 5.55 & 4.89 & 1.84 & $12 \%$ & $62 \%$ \\
\hline Trend & 0.04 & 0.02 & 0.01 & $50 \%$ & $50 \%$ \\
\hline Linemax & 90.7 & 91.25 & 0 & $-1 \%$ & $100 \%$ \\
\hline Trap. time & 30.72 & 25.73 & NaN & $16 \%$ & $\gg 100 \%$ \\
\hline
\end{tabular}

Table 4: Comparison of Recurrence Quantifiers of TTR time-series realized actual PFS data loggers with n-SDE and exponential models, respectively

\begin{tabular}{|c|c|c|c|c|c|}
\hline \multirow{2}{*}{ Metric } & \multicolumn{3}{|c|}{ TTR } & \multicolumn{2}{c|}{ TTR Error \% } \\
\cline { 2 - 6 } & Actual & Model & Exp & Model & Exp \\
\hline Recrate & 43.24 & 21.78 & 0.13 & $50 \%$ & $99 \%$ \\
\hline Determinism & 97.84 & 97.9 & 51.31 & $0 \%$ & $48 \%$ \\
\hline Laminarity & 115 & 102 & 30 & $11 \%$ & $71 \%$ \\
\hline Entropy & 5.71 & 4.65 & 2.38 & $19 \%$ & $49 \%$ \\
\hline Trend & 0.04 & 0.04 & 0.01 & $0 \%$ & $75 \%$ \\
\hline Linemax & 92.08 & 95.32 & 0 & $-4 \%$ & $100 \%$ \\
\hline Trap. time & 33.01 & 29.1 & NaN & $12 \%$ & $\gg 100 \%$ \\
\hline
\end{tabular}

Additionally, the n-SDE model is more likely to capture the underlying nonlinear dynamic patterns such as recurrence [69] of actual operations. Tables 3 and 4 suggest that most of the recurrence quantifiers [69], that measure the extent of dependencies among TBF and TTR processes, estimated from n-SDE model are much closer to those estimated from actual data compared to those from an exponential model. Further investigations are necessary to develop this approach for effective (faster and more accurate) fitting of the downtime distributions and their interrelationships. 


\section{Concluding remarks}

In this paper, we have investigated the application of piecewise affine (constant) differential equation models to capture the coupled dynamics of degradation and restoration processes inherent to industrial machines and other real-world mechanical systems. The piecewise affine structure and the order of the differential equations provide one of the simplest means to capture the salient dynamic behaviors. The model admits a solution that mimics the fairly regular (periodic) long-term breakdown and repair intervals and the highly right skewed shortterm breakdown repair intervals. We have provided results on the local stability and the basin of attraction for a solution of this class of piecewise-affine differential equation models. Under the conditions where periodic solutions are locally stable, the trajectories are found to merge with the periodic orbits within five crossings across the switching surfaces. Furthermore, the existence of a fairly broad basin of attraction enhances the tolerance for lower precision in numerical solvers used for integrating affine equations, i.e., numerical solutions are less prone to finite precision errors and are robust to the tolerance specifications used in the integration routines, the specification of the initial conditions, and finite perturbations to the model parameters. The numerical studies conducted to verify these theoretical results indicate that the model, parametrized to meet the stability and the perturbation criteria specified in the theoretical results, can capture the multimodal behavior of data from a real-world manufacturing system. The short-time TBF and TTR distributions exhibit right-skewed exponential-like characteristics with $\nu$ values fairly consistent with theoretical results.

We have also conducted a case study to integrate the above model into an assembly line segment. Sigmoidal function theory is used to remove discontinuities resulting from the onset and offset of breakdown-restored states. These discontinuities are known to introduce instabilities in the numerical integration routines used to solve the model. The n-SDE modeling approach was tested on an 18 station assembly line segment in Matlab's Simulink environment. The results were compared with those from a real-world production line observed during a one-year period. The results indicate that the n-SDE model can capture the marginal distributions of TBF and TTR better than commonly used exponential distribution models. More pertinently, the model is also able to capture the salient trends in the assembly line dynamics, including the relative throughput losses due to blocking, starving, and machine breakdown, as well as TBF-TTR interactions. An extensive set of metrics was used to compare the statistical and nonlinear dynamical behaviors gathered from the models vs. actual assembly line data. Collectively, these results allude to a straightforward approach to parametrize piecewise affine differential equation models with random 
perturbations to capture the complex degradation and repair dynamics of real-world engineering systems. Our ongoing work is focused on deriving similar results as in Theorems 1-6 for sigmoidal functions and using piecewise nonlinear models with polynomial structures to capture additional nuances of degradation and restoration dynamics in engineering systems.

\section{References}

[1] V. S. Kouikoglou, Y. A. Phillis, Hybrid Simulation Models of Production Networks, Kluwer Academic, New York, 2001.

[2] S. Kachani, G. Perakis, Fluid dynamics models and their applications in transportation and pricing, European Journal of Operational Research 170 (2) (2006) 496 - 517.

[3] V. G. Kulkarni, Fluid models for single buffer systems, in: J. H. Dshalalow (Ed.), Frontiers in queueing, Vol. 7, CRC Press, Inc., Boca Raton, FL, USA, 1997, pp. 321-338.

[4] S. Y. D. Wu, R. A. Wysk, An application of discrete-event simulation to on-line control and scheduling in flexible manufacturing, International Journal of Production Research 27 (9) (1989) 1603-1623.

[5] V. B. Houdt, C. Blondia, Approximated transient queue length and waiting time distributions via steady state analysis, Stochastic Models 21 (2-3) (2005) 725-744.

[6] A. Azaron, H. Katagiri, K. Kato, M. Sakawa, Modelling complex assemblies as a queueing network for lead time control, European Journal of Operational Research 174 (1) (2006) 150-168.

[7] P. R. Kumar, S. P. Meyn, Stability of queuing networks and scheduling policies, IEEE Transactions on Automatic Control 40 (2) (1995) 251-260.

[8] T. Bielecki, P. R. Kumar, Optimality of zero-inventory policies for unreliable manufacturing systems, Operations Research 36 (4) (1988) 532-541.

[9] J. G. Dai, S. P. Meyn, Stability and convergence of moments for multiclass queueing networks via fluid limit models, IEEE Transactions on Automatic Control 40 (11) (1995) 1889-1904.

[10] V. S. Kouikoglou, Y. A. Phillis, Discrete event modeling and optimization of unreliable production lines with random rates, IEEE Transactions on Robotics and Automation 10 (2) (1994) 153-159. 
[11] O. Z. Maimon, S. B. Gershwin, Dynamic scheduling and routing for flexible manufacturing systems that have unreliable machines, Operations Research 36 (2) (1988) 279-292.

[12] S. P. Meyn, Transience of multiclass queueing networks via fluid limit models, The Annals of Applied Probability 5 (4) (1995) 946-957.

[13] S. P. Meyn, Stability and optimization of queueing networks and their fluid models, Lectures in applied mathematics - American mathematical society 33 (1997) 175-200.

[14] J. R. Perkins, P. R. Kumar, Stable, distributed, real-time scheduling of flexible manufacturing/assembly/diassembly systems, IEEE Transactions on Automatic Control 34 (2) (1989) 139-148.

[15] B. Tan, S. B. Gershwin, Analysis of a general markovian two-stage continuous-flow production system with a finite buffer, International Journal of Production Economics 120 (2) (2009) 327-339.

[16] B. Tan, S. B. Gershwin, Modelling and analysis of markovian continuous flow systems with a finite buffer, Annals of Operations Research 182 (1) (2011) 5-30.

[17] M. Colledani, S. B. Gershwin, A decomposition method for approximate evaluation of continuous flow multistage lines with general markovian machines, Annals of Operations Research 209 (1) (2013) 5-40.

[18] S. H. Strogatz, Nonlinear Dynamics and Chaos: With Applications to Physics, Biology, Chemistry, and Engineering, Westview Press, Cambridge, MA, 1994.

[19] H. Yang, S. T. S. Bukkapatnam, L. G. Barajas, Local recurrence-based performance prediction and prognostics in the nonlinear and nonstationary systems, Pattern Recognition 44 (8) (2011) 1834-1840.

[20] T. Altiok, B. Melamed, The case for modeling correlation in manufacturing systems, IIE Transactions 33 (9) (2001) 779-791.

[21] L. Miron, B. Melamed, A. K. Tsiolis, The impact of autocorrelation on queuing systems, Management Science 39 (3) (1993) 322-339.

[22] Y. Dallery, On modeling failure and repair times in stochastic models of manufacturing systems using generalized exponential distributions, Queueing Systems 15 (1) (1994) 199-209. 
[23] G. Rakesh, C. Alka, Analysis of stochastic models in manufacturing systems pertaining to repair machine failure, in: C. T. Leondes (Ed.), Computer-Aided Design, Engineering, and Manufacturing: Systems Techniques and Applications, Optimization Methods for Manufacturing, Vol. IV, CRC Press, 2000.

[24] B. Balciolu, D. L. Jagerman, T. Altiok, Approximate mean waiting time in a gi/d/1 queue with autocorrelated times to failures, IIE Transactions 39 (10) (2007) 985-996.

[25] Y. H. Kim, L. C. Thomas, Repair strategies in an uncertain environment: Markov decision process approach, Journal of the Operational Research Society 57 (8) (2006) 957-964.

[26] L. Cui, W. Kuo, H. T. Loh, M. Xie, Optimal allocation of minimal and perfect repairs under resource constraints, IEEE Transactions on Reliability 53 (2) (2004) 193-199.

[27] E. A. Elsayed, An optimum repair policy for the machine interference problem, Journal of the Operational Research Society 32 (9) (1981) 793-801.

[28] Q. Zheng, X. Hong, S. Ray, Nonlinear resource allocation in restoration of compromised systems, in: IEEE International Conference on Communications, Vol. 1, Istanbul, 2006, pp. 429-434.

[29] S. Han, K. G. Shin, Efficient spare-resource allocation for fast restoration of real-time channels from network component failures, in: The 18th IEEE Real-Time Systems Symposium, San Francisco, CA, 1997, pp. 99-108.

[30] T. Osogami, M. Harchol-Balter, Closed form solutions for mapping general distributions to quasi-minimal ph distributions, Performance Evaluation 63 (6) (2006) 524-552.

[31] K. Linderman, K. E. McKone-Sweet, J. C. Anderson, An integrated systems approach to process control and maintenance, European Journal of Operational Research 164 (2) (2005) 324-340.

[32] J. Sun, M. Rahman, Y. Wong, G. Hong, Multiclassification of tool wear with support vector machine by manufacturing loss consideration, International Journal of Machine Tools and Manufacture 44 (11) (2004) $1179-1187$.

[33] D. Armbruster, S. Gottlich, M. Herty, A scalar conservation law with discontinuous flux for supply chains with finite buffers, SIAM Journal on Applied Mathematics 71 (4) (2011) 1070-1087.

[34] R. Bierbooms, I. J. B. F. Adan, M. V. Vuuren, Performance analysis of exponential production lines with fluid flow and finite buffers, IIE Transactions 44 (12) (2012) 1132-1144. 
[35] J. G. Kimemia, S. B. Gershwin, An algorithm for the computer control of production in a flexible manufacturing system, in: 20th IEEE Conference on Decision and Control including the Symposium on Adaptive Processes, Vol. 20, 1981, pp. 628-633.

[36] J. Kim, S. B. Gershwin, Integrated quality and quantity modeling of a production line, OR spectrum $27(2-3)$ (2005) 287-314.

[37] M. Colledani, T. Tolio, Integrated quality, production logistics and maintenance analysis of multi-stage asynchronous manufacturing systems with degrading machines, CIRP Annals-Manufacturing Technology 61 (1) (2012) 455-458.

[38] M.-H. Shu, B.-M. Hsu, K. C. Kapur, Dynamic performance measures for tools with multi-state wear processes and their applications for tool design and selection, International journal of production research 48 (16) (2010) $4725-4744$.

[39] L. Schruben, Analytical simulation modeling, in: Winter Simulation Conference, 2008, pp. 113-121.

[40] Y. Han, Q. Cao, Y. Chen, M. Wiercigroch, Chaotic thresholds for the piecewise linear discontinuous system with multiple well potentials, International Journal of Non-Linear Mechanics 70 (0) (2015) 145-152, nonlinear Dynamics in Engineering: Modelling, Analysis and Applications.

[41] W. Loud, Branching phenomena for periodic solutions of non-autonomous piecewise linear systems, International Journal of Non-Linear Mechanics 3 (3) (1968) 273 - 293.

[42] P. Casini, F. Vestroni, Characterization of bifurcating non-linear normal modes in piecewise linear mechanical systems, International Journal of Non-Linear Mechanics 46 (1) (2011) 142-150.

[43] S. Natsiavas, S. Theodossiades, I. Goudas, Dynamic analysis of piecewise linear oscillators with time periodic coefficients, International Journal of Non-Linear Mechanics 35 (1) (2000) 53-68.

[44] T. Caughey, A. Vijayaraghavan, Stability analysis of the periodic solution of a piecewise-linear non-linear dynamic system, International Journal of Non-Linear Mechanics 11 (2) (1976) 127-134.

[45] T. Caughey, A. Vijayaraghavan, Forced oscillations of a piecewise-linear non-linear dynamic system with several degrees of freedom, International Journal of Non-Linear Mechanics 12 (6) (1977) 339-353. 
[46] M. Yuichiro, M. Haruki, Piecewise linear model for water column oscillator simulating reactor safety system, International Journal of Non-Linear Mechanics 38 (2) (2003) 213-223.

[47] S. Natsiavas, Dynamics of piecewise linear oscillators with van der pol type damping, International Journal of Non-Linear Mechanics 26 (3) (1991) 349-366.

[48] C.-S. Liu, Two-dimensional bilinear oscillator: group-preserving scheme and steady-state motion under harmonic loading, International Journal of Non-Linear Mechanics 38 (10) (2003) 1581 - 1602.

[49] H.-K. Hong, C.-S. Liu, Internal symmetry in the constitutive model of perfect elastoplasticity, International Journal of Non-Linear Mechanics 35 (3) (2000) 447 - 466.

[50] T. Ushio, K. Hirai, Chaotic behavior in piecewise-linear sampled-data control systems, International Journal of Non-Linear Mechanics 20 (5) (1985) 493-506.

[51] J. P. Kharoufeh, S. M. Cox, Stochastic models for degradation-based reliability, IIE Transactions 37 (6) (2005) $533-542$.

[52] J. P. Kharoufeh, S. M. Cox, M. E. Oxley, Reliability of manufacturing equipment in complex environments, Annals of Operations Research 209 (1) (2013) 231-254.

[53] E. M. Izhikevich, Which model to use for cortical spiking neurons?, IEEE transactions on Neural Networks 15 (5) (2004) 1063-1070.

[54] A. Tonnelier, W. Gerstner, Piecewise linear differential equations and integrate-and-fire neurons: Insights from two-dimensional membrane models, Physical Review E 67 (2) (2003) 021908.

[55] J. Li, G. Lykotrafitis, M. Dao, S. Suresh, Cytoskeletal dynamics of human erythrocyte, Proceedings of the National Academy of Sciences 104 (12) (2007) 4937-4942.

[56] W. Mocek, R. Rudnicki, E. Voit, Approximation of delays in biochemical systems, Mathematical Biosciences 198 (2) (2005) 190-216.

[57] D. A. Edwards, Biochemical reactions on helical structures, SIAM Journal on Applied Mathematics 60 (4) (2000) 1425-1446. 
[58] D. J. Tannor, S. A. Rice, Control of selectivity of chemical reaction via control of wave packet evolution, The Journal of Chemical Physics 83 (10) (1985) 5013-5018.

[59] A. Veliz-Cuba, A. Kumar, K. Josic, Piecewise linear and boolean models of chemical reaction networks, Bulletin of Mathematical Biology 76 (12) (2014) 2945-2984.

[60] U. Mittal, H. Yang, S. T. S. Bukkapatnam, L. G. Barajas, Dynamics and performance modeling of multi-stage manufacturing systems using nonlinear stochastic differential equations, in: CASE 2008 IEEE International Conference on Automation Science and Engineering, 2008, pp. 498-503.

[61] H. K. Khalil, J. Grizzle, Nonlinear systems, Vol. 3, Prentice hall Upper Saddle River, 2002.

[62] J. M. Gonçalves, Regions of stability for limit cycle oscillations in piecewise linear systems, IEEE Transactions on Automatic Control 50 (11) (2005) 1877-1882.

[63] G. Davrazos, N. T. Koussoulas, A review of stability results for switched and hybrid systems, in: Proceedings of 9th Mediterranean Conference on Control and Automation, 2001.

[64] S. Bukkapatnam, M. Malshe, P. Agrawal, L. Raff, R. Komanduri, Parametrization of interatomic potential functions using a genetic algorithm accelerated with a neural network, Physical Review B 74 (22) (2006) 224102.

[65] D. Elliott, Sigmoidal transformations and the trapezoidal rule, ANZIAM Journal 40 (1998) 77-137.

[66] B. I. Yun, An extended sigmoidal transformation technique for evaluating weakly singular integrals without splitting the integration interval, SIAM Journal on Scientific Computing 25 (1) (2003) 284-301.

[67] J. H. Holland, Adaptation in Natural and Artificial Systems: An Introductory Analysis with Applications to Biology, Control, and Artificial Intelligence, MIT Press, Cambridge, MA, 1992.

[68] R. R. Inman, Empirical evaluation of exponential and independence assumptions in queueing models of manufacturing systems, Production and Operations Management 8 (4) (1999) 409-432.

[69] N. Marwan, R. M. Carmen, M. Thiel, J. Kurths, Recurrence plots for the analysis of complex systems, Physics Reports 438 (5) (2007) 237-329.

[70] A. H. Jazwinski, Stochastic Processes and Filtering Theory, Academic Press, New York, 1970. 
[71] A. Papoulis, S. U. Pillai, Probability, Random Variables, and Stochastic Processes, McGraw-Hill, New York, NY, 2002.

[72] S. M. Ross, Introduction to Probability Models, Academic Press, San Diego, CA, 2009.

\section{Appendix A. Proof of Theorem 1}

The constraints in Theorem 1 are proposed to sufficiently guarantee that the system follows the expected trajectory.

The transition state between consecutive segments $j-1$ and $j, j \in 1,2, \ldots, 11$ meets at least one of the following eight switching conditions:

$$
\begin{aligned}
& \beta_{j-1}(t)=\alpha_{1}, \quad \rho_{j-1}(t)=0, \quad-\rho_{j-1}(t)=0, \quad \beta_{j-1}(t)=\alpha_{2}, \\
& \dot{\rho}_{j-1}(t)=\alpha_{3}, \quad \beta_{j-1}(t)=\alpha_{4}, \quad \beta_{j-1}(t)=\alpha_{5}, \quad \dot{\rho}_{j-1}(t)=\alpha_{6} .
\end{aligned}
$$

The times associated with those switching conditions are denoted by $t_{j}^{(i)}, i \in\{1,2, \ldots, 8\}, j \in 1,2, \ldots, 11$. The switching time (time at which dynamics switches) from segment $j-1$ to $j$ is given by

$$
T_{j}=\min _{k \in I_{j}}\left\{t_{j}^{(k)}\right\}
$$

where

$$
I_{j}=\left\{k: t_{j}^{(k)}>T_{j-1}\right\}
$$

The constraints stated in (3) are deemed to enforce a particular switching sequence as detailed in the following exposition. Here, we detail the determination of $T_{j}$ 's and the resulting constraints at segments $j=1$, and outline the procedure with which other constraints were obtained.

Segment 1: The trajectories in this segment follow transient dynamics until the next switching condition is met. The trajectories are chosen to originate from the following initial condition:

$$
\beta(0)=\alpha_{1}, \rho(0)=0, \dot{\rho}(0)=-K_{1}
$$


Under these conditions, $(1,2)$ reduce to

$$
\dot{\beta}(t)=K_{2}, \ddot{\rho}(t)=0 .
$$

And the system trajectories are defined by

$$
\beta_{0}(t)=\alpha_{1}+K_{2} t, \rho_{0}(t)=K_{1}(-t)
$$

Segment 2: Solving for t's in (A.1) we have the following candidates for switching time $T_{1}$ :

$$
\begin{gathered}
t_{1}^{(1)}=t_{1}^{(2)}=t_{1}^{(3)}=0 \\
t_{1}^{(4)}=\frac{-\alpha_{1}+\alpha_{2}}{K_{2}}, t_{1}^{(6)}=\frac{-\alpha_{1}+\alpha_{4}}{K_{2}}, t_{1}^{(7)}=\frac{-\alpha_{1}+\alpha_{5}}{K_{2}} .
\end{gathered}
$$

Also, $t_{1}^{(5)}, t_{1}^{(8)}$ are not admissible, as the corresponding switching conditions violate the dynamics at the boundary as specified in (A.8).

Segment 2 models the degradation of the machine from its peak condition, where when $\beta(t)$ decreases to a certain level $\alpha_{5}$, the restoration- must increase. Therefore, we want the event associated with $t_{1}^{(6)}$ precedes other $t_{1}^{(i)}$ s, i.e., $T_{1}=\min \left\{t_{1}^{(4)}, t_{1}^{(6)}, t_{1}^{(7)}\right\}=t_{1}^{(6)}=\frac{\alpha_{4}-\alpha_{1}}{K_{2}}$. This condition could hold if (3g,3h) are met.

Consequently, the dynamics Eqs. $(1,2)$ reduces to:

$$
\begin{aligned}
\dot{\beta}(t) & =K_{2}, \\
\ddot{\rho}(t) & =0 .
\end{aligned}
$$

Also, for the trajectory within segment 2, the initial conditions are given by:

$$
\begin{aligned}
& \beta\left(T_{1}\right)=\alpha_{4}, \\
& \rho\left(T_{1}\right)=\frac{K_{1}}{K_{2}}\left(\alpha_{1}-\alpha_{4}\right), \\
& \dot{\rho}\left(T_{1}\right)=-K_{1} .
\end{aligned}
$$


and the trajectory within this segment is given by:

$$
\begin{aligned}
& \beta_{1}(t)=\alpha_{1}+K_{2} t \\
& \rho_{1}(t)=K_{1}(-t) .
\end{aligned}
$$

Applying this procedure to all subsequent segments, the constraints in (3) sufficiently allow a periodic solution with 10 switches.

\section{Appendix B. Proof of Theorem 2}

Following Theorem 1, the time between failure (the period of the solution to $(1,2)$ ), time to repair, time to failure and availability are given by:TBF $=T B F=T_{11}-T_{1}, T T R=T_{5}-T_{4}, T T F=T B F-T T R, \Lambda=\frac{T T F}{T B F}$. By substituting formulas for $T_{1}, T_{4}, T_{5}, T_{11}$ derived from the proof of Theorem 1 we have $(5,6,7)$.

\section{Appendix C. Proof of Theorem 3}

$$
\begin{aligned}
& \text { We prove this Theorem based on Proposition 1. Here, } A_{i}=\left(\begin{array}{ccc}
0 & 0 & 0 \\
0 & 0 & 1 \\
0 & 0 & 0
\end{array}\right)=0 \text {, and } A_{i}^{2}=0 \text {. Therefore, } \\
& \qquad e^{A_{i} t_{i}^{*}}=\sum_{k=0}^{\infty} \frac{1}{k !}\left(A_{i} t_{i}^{*}\right)^{k}=E+A_{i} t_{i}^{*}
\end{aligned}
$$

where $E$ is the identity matrix and $t_{i}^{*}=T_{i+1}-T_{i}$

Combining (10) and (C.1) we have $W_{i}=\left(E-\frac{v_{i} C_{i+1}}{C_{i+1} v_{i}}\right)\left(E+A_{i} t_{i}^{*}\right)$, and

$$
W=\Pi_{i \in I} W_{i}, I=\{1, \ldots, 10\}-\{6\}
$$

Note that matrix $W_{6}$ is not included in (C.2), as the system dynamics does not change when the trajectory cuts plane $\mathcal{S}_{6}$.

From (9) and (C.2), it follows that $W=0$, and all eigenvalues $\lambda_{j}^{w}$ are zero and thus lie within the unit circle. Consequently, the periodic solution $x^{*}(t)$ of $(8)$ is locally stable. 


\section{Appendix D. Proof of Theorem 4}

Similar to the method presented in Theorem 1, the constraints (D.1) are proposed to sufficiently guarantee that the system follows the expected trajectory.

$$
\begin{aligned}
& \alpha_{2} \leq \beta_{0} \leq \alpha_{4}, \rho_{0}<0, \dot{\rho}_{0} \geq \alpha_{6} \text { or } \beta_{0} \geq \alpha_{5}, \\
& \beta_{0} \leq \alpha_{1},-\frac{\rho_{0}}{\dot{\rho}_{0}} \leq 0,
\end{aligned}
$$

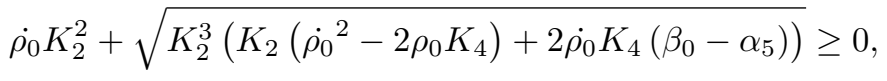

$$
\begin{aligned}
& \alpha_{3} \leq \dot{\rho}_{0}, K_{4}\left(\alpha_{2}-\alpha_{5}\right)+K_{2}\left(\dot{\rho}_{0}-\alpha_{6}\right) \leq 0, \\
& K_{2}\left(-2 \rho_{0} K_{4}+\left(\dot{\rho}_{0}-\alpha_{6}\right)^{2}\right) \\
& +2 K_{4}\left(\beta_{0} \dot{\rho}_{0}-\alpha_{2} \alpha_{6}+\alpha_{5}\left(\alpha_{6}-\dot{\rho}_{0}\right)\right) \leq 0 .
\end{aligned}
$$

Because of (D.1a), (D.1b) is reduced to $\dot{\rho}_{0}<0$. As a consequence, $\left(\dot{\rho}_{0} \geq \alpha_{6}\right.$ or $\left.\beta_{0} \geq \alpha_{5}\right)$ in (D.1a) is reduced to $\beta_{0} \geq \alpha_{5}$. Also,

$$
K_{4}\left(\alpha_{2}-\alpha_{5}\right)+K_{2}\left(\dot{\rho}_{0}-\alpha_{6}\right) \leq 0 \Leftrightarrow \dot{\rho}_{0} \geq \frac{-K_{4}\left(\alpha_{2}-\alpha_{5}\right)}{K_{2}}+\alpha_{6}
$$

On the other hand,

$$
\frac{-K_{4}\left(\alpha_{2}-\alpha_{5}\right)}{K_{2}}+\alpha_{6}-\alpha_{3}=\frac{K_{4}\left(\alpha_{5}-\alpha_{2}\right)+K_{2}\left(\alpha_{6}-\alpha_{3}\right)}{K_{2}} \leq 0
$$

because of $(3 \mathrm{a}, 3 \mathrm{~h})$. Therefore, $\frac{-K_{4}\left(\alpha_{2}-\alpha_{5}\right)}{K_{2}}+\alpha_{6} \leq \alpha_{3}$. It follows that (D.1d) is satisfied if $\alpha_{3} \leq \dot{\rho}_{0}$.

(D.1c) is equivalent to

$$
\begin{gathered}
\sqrt{K_{2}^{3}\left(K_{2}\left({\dot{\rho_{0}}}^{2}-2 \rho_{0} K_{4}\right)+2 \dot{\rho_{0} K_{4}}\left(\beta_{0}-\alpha_{5}\right)\right)} \geq-\dot{\rho_{0}} K_{2}^{2} \\
\Leftrightarrow\left(-\rho_{0} K_{2}+\dot{\rho_{0}}\left(\beta_{0}-\alpha_{5}\right)\right) \leq 0 .
\end{gathered}
$$

(D.2) is satisfied as $\rho_{0}, \dot{\rho}_{0} \leq 0, K_{2} \leq 0, \beta_{0} \geq \alpha_{5}$. Therefore, (D.1) is equivalent to (11). 


\section{Appendix E. Proof of Theorem 5}

The regions defined in Theorem 5 are the basin of attraction on each of the surfaces and are similar for each surface. The idea is to propose the constraints in the form of sufficient conditions such that the system follows the expected trajectory. The method to construct such conditions are similar to the ones presented in Theorem 1.

Let us consider the impact map from surface 6 to 7, the Lipschitz constant $k$, if it exists, is the smallest number $k$ such that

$$
\left(\sqrt{1+\frac{\left(\frac{\Delta\left(\dot{\rho}_{0}\right)\left(\alpha_{4}-\alpha_{5}\right)}{K_{1}}\right)^{2}+2 \frac{\Delta\left(\dot{\rho}_{0}\right)\left(\alpha_{4}-\alpha_{5}\right)}{K_{1}} \Delta \rho_{0}}{\Delta\left(\dot{\rho}_{0}\right)^{2}+\Delta \rho_{0}^{2}}}\right) \leq k .
$$

However, when $\Delta\left(\dot{\rho_{0}}\right)=\Delta \rho_{0}$, the left hand side of (E.1) is reduced to $\sqrt{1+\frac{\left.\left(\frac{\alpha_{4}-\alpha_{5}}{K_{1}}\right)^{2}+2 \frac{\left(\alpha_{4}-\alpha_{5}\right)}{K_{1}}\right)}{2}}>1$. Therefore, the impact map is not contracting. As a consequence, the basin of attraction formulated in Theorem 5 contain the basin of attraction derived using the method in [62].

\section{Appendix F. Proof of Theorem 6}

We first provide the following Lemma towards proving Theorem 6 .

Lemma 1: If $\gamma(t)$ is mean square (ms) continuous process with $|\gamma(t)|>a>0$ a.e. (almost everywhere), then the integral

$$
\int_{T_{4}+(t d i v T B F) \times T B F}^{t} K_{2} H_{+}(\gamma(\tau)) d \tau
$$

exists and is differentiable.

Proof: Let us define $\Delta(x)=\operatorname{sgm}(x, k)-H_{+}(x)$, where $\operatorname{sgm}(x, k)=\frac{1}{1+e^{-k x}}$. It may be noted that

$$
\forall x \in \mathbb{R} \text {, and }|x|>a>0 \text {, we have }|\Delta(x)| \rightarrow 0 \text { uniformly as } k \rightarrow \infty
$$

This is because

- $\forall x>a,|\Delta(x)|=\left|\frac{1}{1+e^{-k x}}-1\right|=\left|\frac{-e^{-k x}}{1+e^{-k x}}\right|<e^{-k x}<e^{-k a} \rightarrow 0$ as $k \rightarrow \infty$.

- $\forall x<-a,|\Delta(x)|=\left|\frac{1}{1+e^{-k x}}-0\right|<\left|\frac{1}{1+e^{k a}}\right| \rightarrow 0$ as $k \rightarrow \infty$.

Now, substituting $x$ with $\gamma(t)$ for some $|\gamma(t)|>a>0$, a.e., in (F.2), it follows that $\forall \varepsilon>0, \exists K \in \mathbb{N}$ such that $|\Delta(\gamma(t))|<\varepsilon, \forall t>0$ and $k>K$. Consequently, $E\left[|\Delta(\gamma(t))|^{2}\right]<E\left[\varepsilon^{2}\right]=\varepsilon^{2}$ as $k>K, \forall t>0$, or $\forall t>0, \operatorname{sgm}(\gamma, k)$ 
converges uniformly in the ms sense to $H(\gamma)$ as $k \rightarrow \infty$. Furthermore, $\operatorname{sgm}(\gamma, k)$ is ms continuous because $\operatorname{sgm}($.$) is$ continuous, and $\gamma(t)$ is ms continuous. Therefore, it follows that $H(\gamma)$ is ms continuous [70] (Theorem 3.3 p. 62). Consequently, the integral (F.1) exists and is differentiable [70] (Corollary 2 p. 67).

\section{Proof of Theorem 6:}

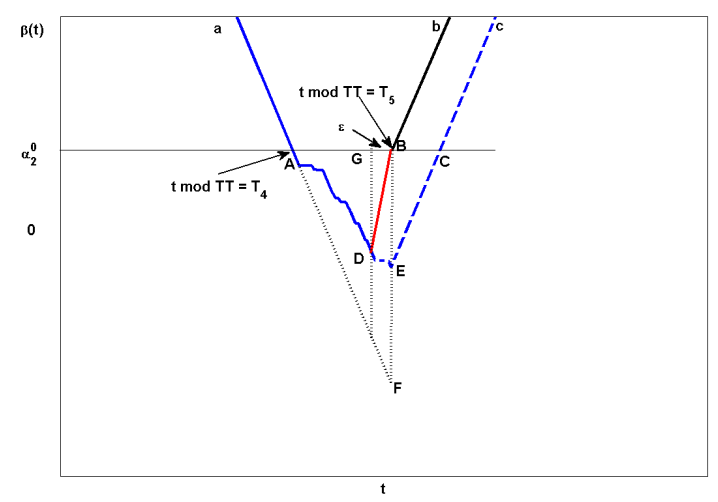

Figure F.7: Diagram of proof of Theorem 6. Without any perturbation, the system evolves as aAGBb. With perturbation to $\alpha_{2}$ and $\epsilon=0$, the system evolves as aADECc. With perturbation to both $\alpha_{2}$ and $K_{2}$, the system evolves as aADBb.

First, consider the random process $\gamma(t)=(-1)^{N(t)}[71]($ p. 379), where $N(t)$ is a Poisson process with parameter $\lambda$, and $N(0)$ is a Bernoulli binary random variable with equal probability for outcomes 0,1 . Evidently, $R_{\gamma \gamma}\left(t_{1}, t_{2}\right)=$ $e^{-2 \lambda\left|t_{1}-t_{2}\right|}\left(t_{1}, t_{2} \geq 0\right)$ is continuous at $(t, t)$. Therefore, it follows from [70] (Theorem $3.2 \mathrm{p}$. 61) that $\gamma(t)$ is an ms continuous process. Also, the time intervals between successive zero crossings of $\gamma(t)$ are the same as those between consecutive events of the Poisson process $N(t)$, and hence they follow an exponential distribution.

Without loss of generality, let us consider the perturbation for the first period, $T_{1} \leq t \leq T+T_{1}$ of the periodic solution in Fig. (1) as in $(12,13)$. Note that similar constructions follow for other periods. Let us consider $\beta(t)$ defined as:

$$
\beta(t)= \begin{cases}\alpha_{2}^{0}+\int_{T_{4}}^{t} K_{2}^{0} H(\gamma(\tau)) d \tau & \text { if } T_{4} \leq t<T_{5}-\varepsilon \\ \alpha_{2}^{0}+\tilde{K}_{2}\left[t-T_{5}\right] & \text { if } T_{5}-\varepsilon \leq t<T_{5} \\ \beta^{0}(t) & \text { otherwise }\end{cases}
$$

where $\beta^{0}(t)$ is the solution of the unperturbed deterministic model in the corresponding region, $\tilde{K}_{2}$ is defined in Eqn. (13). 
To show that $\rho(t)$ satisfies (2), we note from (F.3) that $\beta(t) \leq \alpha_{2}^{0}$ as $K_{2}^{0} \leq 0$ when $T_{4} \leq t<T_{5}-\varepsilon$ in (F.3a) and $K_{2}\left[t-T_{5}\right] \leq 0$ when $T_{5}-\varepsilon \leq t<T_{5}$ in (F.3b). Therefore, when $T_{4} \leq t \leq T_{5}, \beta(t) \leq \alpha_{2}^{0}<\alpha_{5}<\alpha_{4}$, i.e., $\rho(t)$ satisfies (2). Outside this region, $\rho(t)$ automatically satisfies (2) because of its definition.

Let us now verify that $\beta(t)$ defined in (F.3) satisfies (1). From (F.3a) and (12a) we have

$$
\beta(t)-\alpha_{2}(t)=\gamma(t)
$$

Applying Lemma 1 to (F.3a), we have:

$$
\frac{d \beta(t)}{d t}=K_{2}^{0} H(\gamma(t))
$$

It follows from (F.4) and (F.5) that $\frac{d \beta(t)}{d t}=K_{2}^{0} H\left(\beta(t)-\alpha_{2}(t)\right)$ or $\beta(t)$ satisfies (1).

Similarly, from (F.3b) and (12b) we have

$$
\beta(t)-\alpha_{2}(t)=\tilde{K}_{2}\left[t-\left(T_{5}-\varepsilon\right)\right] \geq 0
$$

and

$$
\frac{d \beta(t)}{d t}=\tilde{K}_{2}=\tilde{K}_{2} H\left(\beta(t)-\alpha_{2}(t)\right)
$$

or $\beta(t)$ satisfies (1).

At $t=T_{5}, \alpha_{2}\left(T_{5}\right)=\alpha_{2}^{0}, \beta\left(T_{5}\right)=\alpha_{2}^{0}, \rho\left(T_{5}\right)=\rho_{5}\left(T_{5}\right), \rho^{\prime}\left(T_{5}\right)=\rho_{5}^{\prime}\left(T_{5}\right)$. When $t \rightarrow T_{5}^{+}, \beta^{\prime}(t)=\beta_{5}^{\prime}(t)$ and $\rho^{\prime \prime}(t)=\rho_{5}^{\prime \prime}(t)$. Therefore, the system dynamics does not change outside $\left[T_{4}, T_{5}\right)$.

Furthermore, as $\beta(t)-\alpha_{2}(t)=\gamma(t)$ for $T_{4} \leq t<T_{5}-\varepsilon$, it may be noted that the zero crossing time-points of $\gamma(t)$ and $\beta(t)-\alpha_{2}(t)$ are identical. As as result, the interval between the $i^{t h}$ and $(i+1)^{t h}$ zero crossing of $\beta(t)-\alpha_{2}(t)$ denoted by $\tau_{i}$ are independent and follows exponential distribution with mean interval length of $\frac{1}{\lambda}$.

Case 1: the first zero results from a down crossing and TTRs: $\tau_{1}, \tau_{3}, \ldots$ follow exponential distribution.

Case 2: the first zero results from an up crossing and TTRs: $\tau_{2}, \tau_{4}, \ldots$ follow exponential distribution.

In both cases, TBF: $\tau_{1}+\tau_{2}, \tau_{3}+\tau_{4}, \ldots$ follow Erlang distribution with parameters $(2, \lambda)[72]$. Thus, TTRs follow the exponential distribution with skewness of 2, and TBFs follow the Erlang(2) distribution with skewness of $2^{1 / 2}$. 


\section{Appendix G. Proof of Corollary 1}

Note that the result in Theorem 6 is applied for each period of the periodical solution in Theorem 1. In each period, by replacing $\alpha_{2}, K_{2}$ in the deterministic model $(1,2)$ with $(12,14)$, there are multiple short TBF which exhibit right skewed distribution as shown in the Theorem 6. Together with long TBF from the deterministic model $(1,2)$, we have a multimodal distribution. 